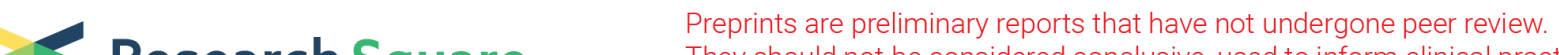 Research Square They should not be considered conclusive, used to inform clinical practice, or referenced by the media as validated information.
}

\section{Transcriptome and targeted metabolome reveal the regulation network of Lilium davidii var. unicolor during dormancy release}

\section{Xinyue Fan}

Shenyang Agricultural University

\section{Yue Yang}

Shenyang Agricultural University

\section{Min Li}

Shenyang Agricultural University

\section{Linlan Fu}

Shenyang Agricultural University

\section{Yuqing Zang}

Shenyang Agricultural University

\section{Chunxia Wang}

Shenyang Agricultural University

\section{Tianyou Hao}

Shenyang Agricultural University

Hongmei Sun ( $\nabla$ hmbh@sina.com )

Shenyang Agricultural University https://orcid.org/0000-0003-0800-9539

\section{Research article}

Keywords: Transcriptome, targeted metabolome, ABA, GA4, carbohydrate metabolism, TF family

Posted Date: December 16th, 2020

DOI: https://doi.org/10.21203/rs.3.rs-126184/v1

License: (c) (1) This work is licensed under a Creative Commons Attribution 4.0 International License. Read Full License 


\section{Abstract}

Background $邓$ Regulation and control of bulb dormancy are critical for ensuring annual production and high-level cultivation of lily. Application of low temperatures is the most effective method for breaking lily bulb dormancy, but the molecular regulatory mechanism underlying this response is unclear.

Results囚Herein, targeted metabolome and transcriptome analyses were performed with buds of Lilium davidii var. unicolorbulbs stored for 0,50 and 100 days at $4^{\circ} \mathrm{C}$. Dormancy release mainly depended on the accumulation of $\mathrm{GA}_{4}$ and $\mathrm{GA}_{7}$, which are synthesized by the "non13-hydroxylation" pathway, rather than $\mathrm{GA}_{3}$, and $A B A$ was degraded in the process. The contents of nonbioactive $G A_{9}, G_{15}$ and $G A_{24}$, the precursors of $\mathrm{GA}_{4}$ synthesis, increased with bulb dormancy release. Altogether, 113,252 unique transcripts were de novo assembled through high-throughput transcriptome sequences, and 639 genes were continuously differentially expressed. Enrichment analysis showed that the carbohydrate metabolism and hormone metabolism pathways play major roles in dormancy release. Energy sources during carbohydrate metabolism mainly depend on glycolysis and the PPP pathway rather than on the TCA cycle. Association analysis of the transcriptome and target metabolome showed that genes related to ABA, GA, starch and sucrose metabolism and some TF families, such as MYB, WRKY, NAC and TCP, involved in dormancy release were highly correlated with the target metabolome. Coexpression analysis further confirmed that $A B I 5, P Y L 8, P Y L 4$ and $P P 2 C$, vital ABA signaling elements, regulated GA3ox and GA20ox in the $\mathrm{GA}_{4}$ biosynthesis pathway. The TFs WRKY32, WRKY71, MYB, DAM14, NAC8, ICE1, bHLH93 and TCP15 also participated in the $\mathrm{ABA} / \mathrm{GA}_{4}$ regulatory network, and ICE1 may be the key factor linking temperature signals and hormone metabolism.

Conclusions囚These results will help to reveal the bulb dormancy molecular mechanism and to develop new strategies for high-quality bulb production.

\section{Background}

Dormancy is a phenomenon in which the growth and metabolism of plants or their organs are temporarily suspended during a certain period of development. It is a powerful adaptation formed by plants through a long-term evolutionary process to resist external adverse environments and maintain the continuation of the species [1]. Compared with seeds and buds, the dormancy of abnormal organs is more complex, and research progress on this phenomenon has been relatively slow. Lily, as an ancient cosmopolitan flower, has high ornamental and edible value [2]. Physiological dormancy exists in most species of lily, and the dormancy degree varies greatly among species, so it is an excellent material for studying the dormancy of abnormal organs. To date, low-temperature treatment is still the most effective method to break dormancy. Most of the previous studies have focused on physiological, biochemical and morphological changes [3], but there are few studies on the molecular mechanisms underlying these changes. Low-temperature storage, without theoretical support, is uninformed, inefficient and costly. Therefore, it is of great significance to elucidate the molecular mechanism of lily bulb dormancy for 
rational utilization and regulation of dormancy to facilitate the production of high-quality bulbs and to realize annual production of bulbs and cut flowers.

During the process of plant dormancy release, carbohydrate metabolism is vigorous and provides energy for later germination. The starch content in the bulbs of Lilium pumilum decreases with prolonged lowtemperature storage time [3]. In potatoes, a-amylase induces tuber germination by hydrolysis of soluble plant glycogen. Starch decreases and sucrose accumulates in kiwifruit and cherry during dormancy release $[4,5]$, indicating that carbohydrate metabolism is a common feature in plants. In addition, hormones respond to temperature signals to regulate plant dormancy [2]. At the early stage of dormancy, growth stagnation is closely related to the decrease in GA levels. With the extension of storage time, GA levels continue to rise sharply, and dormancy is gradually lifted [6]. GAs can replace the function of chilling, strengthen the activity of 1,3-glucanase, and promote the hydrolysis of callose during bud dormancy release in Populus [7]. The antagonism between ABA and GA/CK, which is considered to be the key factor of dormancy, has also been revealed in plants with typical reproductive organs such as Solanum tuberosum L. [8], Zantedeschia [9] and Gladiolus hybridus [10]. ABA controls the dormancy and germination processes by regulating the expression of starch and sucrose metabolism genes such as AtAMY3, AtBAM1, VvSSs1, VvSSs3 and VVINV in Arabidopsis thaliana [11] and grape [12]. In Arabidopsis thaliana, AtGASA6, a gene involved in gibberellin biosynthesis, is a key factor involved in gibberellin, abscisic acid and carbohydrate metabolic pathways and interacts with RGL2 and AtEXPA1 to regulate seed germination [13].

A number of transcription factors (TFs) involved in dormancy regulation have also been identified, including the WRKY, MADS-Box, bZIP, TCP and other TF families. OsWRKY71 and OsWRKY51 jointly participate in $\mathrm{GA}$ and $\mathrm{ABA}$ signaling pathways, controlling the activity of a-amylase and regulating seed dormancy [14]. The transcriptional activities of PpDAM1 and PpDAM3 in Pyrus pyrifolia were activated by $P p C B F$ and negatively regulated PpFT2, which induced bud dormancy [15]. AtTCP14 and AtTCP15 were negatively regulated by GAI and RGL2, members of the DALLA protein family in the gibberellin signaling pathway, respectively, and inhibited seed germination by affecting cell proliferation [16]. The expression of PpyDAM3 in Asian pears is affected by ABA levels and is negatively regulated by PpyABF2 in association with $A B A$ signaling, which affects bud dormancy [17]. Both AtMYBC1 and AtAP2 are involved in ABA-mediated seed germination [18]. Transcriptome sequencing is a technique to accurately interpret the transcriptional level of plant genes, and it is also one of the most effective tools to excavate target genes. In gladiolus [10] (Gladiolus hybridus), lily [3] (Lilium pumilum), aerial garlic [18], Polygonatum kingianum Coll. et Hemsl rhizomes [19], peanut [20] (Arachis hypogaea L.) and cherry [21] (Prunus avium L.), previous studies on dormancy germination using transcriptome analysis have made significant progress. However, few in-depth analyses of dormancy have been reported by combining transcriptomics with targeted metabolomics.

Previous studies by our group have shown that meristematic cells in lily buds can only divide when they are subjected to low temperature. The bud is the center of bulb dormancy, and rapid germination and orderly growth are morphological indicators of dormancy release. In our research, high-throughput RNA- 
seq was adopted to characterize the mRNA information at the stages of deep dormancy (DD, $0 \mathrm{~d}$ ), middle dormancy (MD, $50 \mathrm{~d}$ ) and dormancy release (DR, $100 \mathrm{~d}$ ). Moreover, dormancy-related pathways dominated by carbohydrate metabolism, hormone metabolism and hormone signal transduction were identified, and key differentially expressed genes (DEGs) were explored. In addition, the levels of several endogenous hormones involved in the response to low temperature were quantitatively detected. By analyzing these correlations, the key DEGs and TFs were screened to construct a correlation network. Our study sheds new light on the molecular mechanism of dormancy in bulbous plants.

\section{Results}

\section{Changes in endogenous hormone contents during dormancy release}

Tests show that a dormant lily bulb needs to be treated at low temperature for $100 \mathrm{~d}$ for dormancy to be broken. We found that in the DR stage, the length of buds increased significantly, the germination rate reached $100 \%$, and the buds grew well, while the deep dormancy and middle dormancy bulbs did not germinate (Fig. 1). Therefore, the determination of hormone contents was carried out at the stages of deep dormancy (DD, $0 \mathrm{~d}$ ), middle dormancy (MD, $50 \mathrm{~d}$ ) and dormancy release (DR, $100 \mathrm{~d}$ ). The contents of $\mathrm{GA}_{4}$ and $G A_{7}$ with biological activity increased notably, while the $\mathrm{GA}_{1}$ and $\mathrm{GA}_{3}$ contents did not change markedly, and the level of $\mathrm{GA}_{3}$ was very low. Nonbioactive $\mathrm{GA}_{9}, \mathrm{GA}_{15}$ and $\mathrm{GA}_{24}$, the precursors of $\mathrm{GA}_{4}$, were increased in the late stage of storage. The same trend was found for $G A_{14}$, while the levels of $G A_{5}$, $\mathrm{GA}_{6}$ and $\mathrm{GA}_{8}$ showed a downward trend. The contents of $\mathrm{GA}_{44}$ and $\mathrm{GA}_{51}$ showed no significant changes (Fig. 2).

The ABA content dropped sharply, especially at the DD-MD stage. In general, the level of IAA showed a slight decrease from DD-MD but reached a peak in the DR stage. The JA content showed a decline at DDMD and increased slightly at MD-DR, which was the same trend observed for SA. The MEJA content increased observably in the late stage. The level of MESA showed a continuous decreasing trend (Fig. 2).

\section{Transcriptome sequencing, de novo assembly, and functional annotation}

To better understand dormancy molecular mechanisms, we performed transcriptome analysis using RNASeq on buds (DD, MD, DR stages), and 9 cDNA libraries were constructed. As shown in Table S1 (Additional file 1), after removal of joints and low-quality sequences, the Q30 base percentage was above $90 \%$, and the GC value was approximately $50 \%$. Since no reference genome is available for lily, Trinity was used to splice clean data. We de novo assembled these reads into 305034690 unique transcripts and 111595541 unigenes, with an N50 length of $1753 \mathrm{bp}$ and an average length of $1441 \mathrm{bp}$. Unigene sequences were compared and annotated with seven database respectively.

\section{Identification of differentially expressed genes during dormancy release}

To identify the DEGs, we performed cross analysis on the gene expression levels in the bud at three stages with a $\mid \log 2$ (fold-change) $\mid>1$ and FDR $<0.05$ as the standard thresholds. For the DD-MD stages, 
we detected a total of 3435 DEGs in buds. Similarly, we identified the DEGs from MD to DR and obtained a total of 10492 DEGs. Obviously, with prolongation of the treatment time, the changes in the buds were more drastic. Comparing DD with DR, we found 6843 DEGs. Next, we compared the DEGs identified in DDMD, MD-DR and DD-DR to pinpoint the common DEGs. Finally, 639 genes (enriched in carbohydrate metabolism, response to stress, hormone metabolism and transcriptional regulation, etc.) were screened for sustained differential expression, indicating their close correlation with dormancy release (Additional file 3: Fig. S1).

Like functional genes, transcription factors also involved in the regulation of various biological processes in plants. Therefore, our analysis also focused on differentially expressed TFs (Table 1), including the MYB, AP2/ERF, NAC, bHLH, WRKY, bZIP, MADS-Box, B3 families, etc. Most of these transcription factors were upregulated in the late stage.

\section{Pathway and biological process (BP) analysis during dormancy release}

To further investigate the biological function of DEGs during dormancy release, all DEGs were mapped in the KEGG database. The results showed that 106,117 and 118 pathways were significantly enriched at the DD-MD, MD-DR and DD-DR stages, respectively. It is worth noting that the DEGs were most enriched in starch and sucrose metabolism, plant hormone signal transduction, amino sugar and nucleotide sugar metabolism, fructose and mannose metabolism, phenylpropanoid biosynthesis, alpha linolenic acid metabolism and 20 other pathways (Additional file 3: Fig. S2 A, B, C).

To better understand the functions of the DEGs, GO enrichment analysis was performed. We noticed that $\mathrm{GO}$ items were mostly concentrated in the biological process and molecular function categories (Additional file 3: Fig. S2 D). We focused on the entries in the biological process (BP) category and found that they were basically consistent with the KEGG enrichment results.

\section{DEGs in response to carbohydrate metabolism}

Carbohydrate metabolism, as an energy source for photosynthetic organ development, is closely related to the dormancy and germination of lily bulbs. A total of 1,604 genes were enriched in carbohydrate metabolism-related pathways. Carbohydrate metabolism is a large and complex biological process, in which starch and sucrose metabolism play a vital role and account for a larger proportion than other pathways, followed by the glycolysis/gluconeogenesis pathway (Fig. 3).

The metabolic balance of starch and sucrose is considered as one of the markers of lily dormancy. In this study, a total of 14 differentially expressed SuSy genes were screened, most of which were upregulated during the whole period. The expression of UGP2 (c-47386.18474 and c-47386.25812) genes was upregulated. The FPKMs of INV (c-47386.11119, C-30693.0, c-39108.0, c-47386.14773 and c47386.41633) and the sucrose phosphate synthase gene SPS (c-47386.1907) were low but significantly upregulated in the late stage of dormancy release. We also found that the expression of Scrk (c-28337.0, c-47386.29767 and c-47386.36397) was downregulated in the early stage but upregulated in the late 
stage. AGPase and SSs, key genes in the starch metabolic pathway, were significantly upregulated in the late stage of low-temperature storage. The amylase gene $A M Y(\mathrm{c}-47386.12073)$ was slightly downregulated in the early stage but upregulated later. The expression levels of DEGs enriched in the galactose metabolic pathway were generally low but were generally upregulated (Fig. 4).

During carbohydrate metabolism, there were three main pathways involved in energy metabolism: glycolysis/gluconeogenesis (EMP), the TCA cycle and the PPP process. Starch phosphorylation occurs at the beginning of the glycolysis pathway, and the DEGs encoding PGM (c-47386.3243 and c47386.28774) were significantly downregulated in the early stage but were then upregulated, similar to the key PK genes (c-47386.37942, c-47386.12488 and c-47386.46122). With the release of dormancy, the upregulated genes accounted for a large proportion of the glycolytic pathway. In the PPP pathway, the ATP-dependent genes RPE (c- 47386.19820), rpiA (c- 47386.19620 and c- 47386.42374) and RPRS (c47386.23352) were upregulated in the late stage.

\section{Hormone metabolic and signal transduction related DEGs}

\section{Hormone synthesis and metabolism}

The regulatory effects of plant endogenous hormones on dormancy have been widely studied. Ten DEGs were the key genes encoding enzymes and were involved in the metabolism of various hormones (Fig. 5A). Both NCED (c-47386.24866 and c-47386.6656) and XERICO (c-47386.19994 and C-47386.22341), genes participating in ABA biosynthesis, were downregulated, showing the same trend as CYP707A (c47386.25413 and c-47386.30266). In contrast, the expression of GA20ox (c-47386.7100 and c47386.44330) and GA3OX (c-47386.12758, c-47386.43545 and c-50304.0) was significantly upregulated. The gibberellin-responsive stimulus protein gene GAST1 (c-47386.14948) was filtered, and its expression was downregulated in the early stage but increased in the later stage. We found that the JA biosynthesis genes LOX (c-33396.0 and c-47386.42989), AOS (c-47386.21748 and c-47386.21642) and OPR (c47386.44259) were significantly upregulated in response to abiotic stress, and the same expression trend was also observed for the SA biosynthesis gene PAL (c-47386.38306).

\section{Hormone signal transduction}

Plant hormone signaling is one of the main factors regulating dormancy and germination. As shown in Fig. $5 \mathrm{~B}$, the highest number of DEGs was involved in ABA signaling, followed by GA signaling. Eight DEGs were the key candidate genes involved in multiple hormone signaling pathways. DELLA (c47386.21970 and c-47386.8637), AUX1 (c-47634.13379) and ARF (c-47634.30204 and c-47634.21786), which belong to the GA and IAA signaling pathways, were significantly upregulated in the later stage. However, the key DEGs of the SA and ETH signaling pathways showed the opposite trend. We found that $B R / 1$ (c-47386.10944 and c-47386.39565) was the only gene that was continuously upregulated throughout the whole process. In the ABA signaling pathway, PYR/PYLs (c-47386.13974, c-47386.42509, c-47386.37128, c-47386.18322, c-47386.6484 and c-47386.43885), the genes encoding receptor proteins, were significantly upregulated. The differences between SnRK2 (c-47386.17917 and c-47386.48342) and 
PP2C (c-47386.47371, c-47634.47372, c-47386.47373, c-29047.0 and c-47386.27169) were significant, showing completely opposite trends.

\section{Correlation analysis between transcripts and the target metabolome}

To understand the regulatory network, we analyzed the correlation between the transcripts at DD vs DR and the targeted metabolome. The unannotated DEGs in the transcriptome results were removed, and the correlation between DEGs and hormone contents was calculated by using the "Spearman" algorithm. Fig. 6 shows an overview of DEGs and endogenous hormones (positive correlation of 1, negative correlation of -1). The correlation heat map shows that $\mathrm{ABA}, \mathrm{GA}_{4}, \mathrm{GA}_{9}$ and $\mathrm{GA}_{24}$ were highly correlated because most of the TFs and DEGs associated with them were common. After screening the correlation results, it was found that DEGs significantly related to endogenous hormones were mostly concentrated in ABA-, GA-, starch- and sucrose-related pathways. Among the many TF families related to dormancy, the WRKY, MYB, MADS-box, NAC and bHLH families were all highly correlated with endogenous hormones. The above results further prove that the process of dormancy release involves not only the change of a certain pathway but also the interaction of multiple pathways.

\section{Key DEG and TF correlation network}

The possible interaction between key TFs and DEGs was further clarified in the data based on llog2 (foldchange)|>2 as the standard, and the gene correlation network was constructed. The genes related to TFs, ABA, GA and the sucrose metabolism pathway are represented in different colors (Fig. 7).

\section{qRT-PCR validation of transcriptome data}

As shown in Fig. 8, 20 DEGs were randomly selected from transcriptional data for QRT-PCR validation in this experiment: 3 ABA signal transduction-related genes. 3 ABA genes related to biosynthesis and metabolism; 3 GA biosynthesis-related genes; 4 genes related to the starch sucrose metabolism pathway and 6 TFs. mRNA-seq and qRT-PCR data were closely related, and the expression of DEGs was basically consistent. The sequencing results showed high accuracy.

\section{Discussion}

\section{Measurement of endogenous hormones}

Dormancy release induced by low temperature is a complex physiological process regulated by various plant hormones [22]. Endogenous IAA, GA and ABA play an important role in bulb dormancy release. The process of dormancy is related not only to the absolute contents of various internal hormones but also to the balance between each hormone [23].

GAs play a vital role in the dormancy of perennial plants. In general, the gibberellin biosynthesis pathway can be divided into two categories: the $\mathrm{GA}_{4}$ and $\mathrm{GA}_{3}$ pathways. $\mathrm{C}_{19}$-gibberellin $\mathrm{GA}_{4}$ is synthesized through 
the "non13-hydroxylation" pathway, and the level of $\mathrm{GA}_{4}$ was increased in the DD-MD stage. Both $\mathrm{GA}_{15}$ and $\mathrm{GA}_{9}$, which also showed increased contents, are the precursors of gibberellin synthesis by $\mathrm{GA}_{4}$. Other bulbous plants, including potatoes [24], gladiolus [25], tulips [26] and garlic [18], are dominated by $\mathrm{GA}_{3}$ pathway. This discovery lays a foundation for the in-depth exploration of the molecular mechanism by which GAs participates in regulating the dormancy of lily bulbs. The antagonistic regulation of $A B A$ and GA plays a central role in the dormancy of lily and is the key to germination. In this experiment, the ABA content of dormant bulbs was the highest and decreased sharply with low-temperature storage. In addition, the ABA/GA ratio showed a downward trend over time. All of the above results indicated that ABA inhibited bulb germination, and a high concentration of GA was a necessary condition for releasing dormancy.

GAs can also effectively enhance the racemase activity required by l-tryptophan to promote the synthesis of IAA [22]. The experimental results showed that the variation trend of the IAA level was consistent with that of GAs during the process of dormancy release, indicating that IAA could coordinate with GAs to regulate dormancy and promote germination. JA was originally isolated from growth inhibitors. It is similar to ethylene and has the effect of promoting aging and delaying growth [27]. In this experiment, the JA content decreased with low temperature storage, while the content of the degradation product MEJA increased gradually, suggesting that JA is a negative regulator of bulb germination of lily.

\section{Pathways Involved In Dormancy Release Carbohydrate metabolism processes}

Starch and sucrose metabolism are important for dormancy release in lily. In this study, the starch and sucrose metabolism pathways were enriched by the most DEGs and had a high correlation with the targeted metabolome. For example, one hexokinase gene, $H K$, and three significantly upregulated fructose kinase genes, Scrks, participate in the plant sugar signaling system. Both the sucrose synthase gene SuSys and the cell wall invertase genes INVs were involved in the hydrolysis of sucrose, and all 12 SuSys genes were markedly upregulated in the late stage. In addition, the sucrose-phosphate synthase (SPS) gene was continuously upregulated. F-6-p could synthesize sucrose through sucrose phosphate synthase, resulting in the sucrose content gradually increasing in the process of dormancy release. Sucrose not only provides energy and raw materials for later germination but is also a key signal molecule involved in cell metabolism during the regulation of dormancy [28]. AGPase is a rate-limiting enzyme gene that converts GIP and ATP into ADPG. Both genes encoding SSs and AGPase enzymes involved in amylopectin synthesis were upregulated in the DD-MD stage. These results suggest that the dormancy release of lily bulbs may be a dynamic process regulated by multiple enzyme genes, including not only the mutual transformation of starch-sucrose but also by a series of energy transformations. EMP, TCA cycle and PPP are the three processes of plant respiration, and they are also the main sources of ATP during carbohydrate metabolism. In the dormancy release of lily bulbs, most of the DEGs were enriched in the glycolytic pathway, and most of the genes were upregulated in the ATP-dependent step, indicating 
that the glycolytic pathway was activated. Previous studies have shown that the release of dormancy in the buds and seeds of perennials is associated with the activation of the PPP pathway. This study showed that the genes related to the PPP pathway were also upregulated in the late storage period, and there was no significant difference in the expression of DEGs involved in the TCA cycle pathway. Therefore, the dormancy release of lily bulbs in $v$ was mainly dependent on glycolysis and PPP pathways, while that of Lilium pumilum DC. Fisch. was mainly dependent on the TCA cycle [3], suggesting that interspecific differences led to significant differences in energy metabolism pathways.

\section{Aba And Ga Related Structure Degs}

According to the combined analysis of the transcriptome and targeted metabolome, we suggest that hypothermia-induced dormancy release is mainly caused by endogenous $G_{4}$ accumulation and $A B A$ degradation. In particular, the ABA biosynthesis rate-limiting enzyme gene NCED may play an essential role in this process. The heterotopic expression of TaNCEDs and TaCYP707A1 altered ABA levels and dormancy in Arabidopsis thaliana [29]. The dynamic balance of StNCED and StCYP707A at the transcriptional level influences the synthesis and degradation of $A B A$ and thus regulates the dormancy of potato tubers [30]. Our results showed that $\angle d N C E D$ was significantly downregulated with the extension of cryopreservation, leading to a decrease in $A B A$ biosynthesis. Moreover, the $\mathrm{GA}_{4}$ biosynthesis genes $\mathrm{GA}_{30 \mathrm{x}}$ and $\mathrm{GA}_{20 \mathrm{ox}}$ were significantly upregulated, $\mathrm{GA}_{4}$ biosynthesis was increased, and bulb dormancy was gradually relieved. In addition, we observed continuous upregulation of the gibberellin stimulus response protein GAST1 and continuous downregulation of three XERICOs, zinc-finger protein genes. GAST1 is involved in GA biosynthesis, and XERICOs are involved in ABA biosynthesis [31, 32]. We further hypothesized that $\mathrm{GA}_{4} / \mathrm{ABA}$ antagonism controls the dormancy and germination of lily bulbs. In recent years, an increasing number of studies have focused on GA and ABA signaling regulatory networks at low temperatures to clarify the molecular mechanism of temperature-mediated seed dormancy and germination [33]. For example, as a key factor connecting $A B A$ and GA signaling pathways, $A B / 4$ inhibits ABA degradation by inhibiting the expression of CYP707A1 and CYP707A2 and promotes GA degradation, leading to seed dormancy in Arabidopsis thaliana [34]. $A B I 5$ is also a key regulator of $A B A$ and GA signaling and is crucial in plant dormancy. For example, $A B / 4$ and $A B / 5$ directly combine with promoters of $G A 2_{o x}$ to regulate seed dormancy in sorghum [35]. GhABI5 functional silencing can promote bulb germination in gladiolus [10]. The ABC transporter PED3 influences the PGIP protein by inhibiting AtABI5 expression, thus promoting seed germination in Arabidopsis thaliana [36]. In this study, a differentially expressed $L d A B I 5$ was obtained, but no $A B I 4$ or homologous genes were found. Coexpression results showed that $\angle d A B I 5$ expression was significantly negatively correlated with $G A 30 X$ and GAST1 but not significantly correlated with GA2Ox. In addition, the expression level of $P Y L$, a major positive regulator of the $A B A$ signal transduction pathway, was significantly upregulated during cryopreservation, while the expression level of $P P 2 C$, a negative regulator, was continuously downregulated. The coexpression network showed that $L d P Y L 4$ and $L d P Y L 8$ were directly related to $\angle d A B I 5$ and $\angle d P P 2 C$, as well as $L d G A 20 o x, L d G A S T 1$ and $L d D E L L A$. This indicates that GA and ABA are 
closely related and complex regulatory networks. The DELLA protein, the core negative regulator of the GA signaling pathway, is directly related to the ABA signaling pathway [37]. NUCLEAR factor-YC (NF-YC3, 4, and 9) of Arabidopsis thaliana interacts with RGa-like2 (RGL2), a member of the DELLA family, to form the NFYC-RGL2 module, which targets $A B I 5$ and regulates the expression of GA- and ABA-related response genes during seed germination [38]. In this study, three DELLA members with upregulated expression during dormancy release were obtained. The GA/ABA molecular regulatory network is likely to directly control the dormancy and germination of lily bulbs.

\section{Tfs Involved In Dormancy Release}

Based on the combined analysis of transcriptomes and the targeted metabolome, we suggest that lily bulb dormancy is directly related to some key TFs, including the WRKY, MYB, NAC, ADS-Box and TCP gene families. In recent years, a growing number of TF families have been shown to be involved in plant dormancy and germination. For example, $A B / 3$ and $A B / 5$ are necessary precursors for $A B A$-induced AtWRKY2 expression, while AtWRKY2 knockout mutant seeds show delayed germination under ABA treatment. This suggests that $A t W R K Y 2$ plays a negative regulatory role in ABA-mediated dormancy maintenance in Arabidopsis thaliana, and AtWRKY40, AtWRKY18, AtWRKY60 and AtWRKY63 also have similar functions [39]. OsWRKY51 and OsWRKY71 are key regulators that mediate GA and ABA cross-talk in wheat aleurone cells and embryonic cells [14]. However, studies on the functional dormancy of the WRKY family have not been reported in Lilium. Our transcriptional results showed that WRKY32 and WRKY71 were significantly different in the MD-DD stage and were strongly correlated with ABA, GA and the sucrose metabolic regulatory network. Thus, these networks are worthy of further study (Fig. 8). The MYB family member AtRSM1 interacts with $\mathrm{HY} 5 / \mathrm{HYH}$ and directly combines with the $A t A B I 5$ promoter to upregulate its expression and then regulate the germination of Arabidopsis seeds [40]. AtPYL9 and AtPYL8 interact with AtMYB77 and AtMYB44 to regulate the expression of downstream genes through the auxin pathway, thus affecting the growth of the Arabidopsis root system [41]. We obtained a total of 43 MYB family members that were significantly differentially expressed. Previous studies have shown that the NAC family is involved in regulating multiple hormone signaling pathways. GhNAC83 affects the dormancy of gladiolus bulbs by regulating ABA signal transduction and CTK biosynthesis [42]. The function of MADs-Box (DAM) is widely studied in perennials such as apples and pears. MADs-Box is upregulated in winter, which is closely related to bud dormancy $[15,43]$. The SOC1 members of the DAM family participate in the dormancy and flowering of apricot [44]; AtSOC1 has also been linked to floral organ development and early flowering [45]. Recent studies have shown that PpTCP20 negatively regulates the expression of $P p D A M 5$ and $P p D A M 6$ to regulate the internal dormancy of peach blossom buds [46]. By inhibiting ABA biosynthesis, activating CK signal transduction and inducing cyclin expression, GhTCP19 promotes the dormancy release of gladiolus bulbs [47]. The bHLH transcription factor family regulates many biological processes. ICE1, a bHLH family member, is a downstream target gene of the GA signaling pathway DELLA protein, which binds to $A B I 5$ to maintain steady-state $A B A$ signaling for Arabidopsis seed germination [48]. We obtained two differentially expressed ICE1 genes, which can positively regulate the expression of GA3ox and negatively regulate the expression of $A B A$ 
signals and biosynthesis-related $A B I 5$ and $N C E D$, indicating that temperature signals may be involved in $A B A / G A_{4}$ regulatory networks; however, it is necessary to further explore their interaction mechanism. The above results indicate that temperature signals may be involved in the $A B A / G A_{4}$ regulatory network, and it is also necessary to further explore this interactive mechanism.

\section{Conclusion}

The purpose of this study is to reveal the mechanism of lily dormancy at low temperature from the perspective of the transcriptome and metabolome. The bulbs of Lilium davidii var. unicolor. stored at $4{ }^{\circ} \mathrm{C}$ were released from dormancy on the 100th day after storage. During this process, endogenous hormone contents changed significantly. Transcriptome sequencing and targeted metabolomics showed that starch and sucrose metabolism, hormone anabolism and signal transduction pathways played important roles in the process of dormancy release. A large number of DEGs were also explored: GA200X, GA3ox, GAI, NCED, PYL4, PYL8 and $A B I 5$ are key genes involved in hormone metabolism and signal transduction pathways, and these genes may be simultaneously regulated by ICE1, WRKY71, R2R3-MYb, DAM2, DAM14, NAC8 and TCP TF families. The strong correlation between sucrose metabolization-related DEGs and the above genes also indicates that there is a large regulatory network involved in the process of dormancy release. In general, the results of this study contribute to a better understanding of the molecular mechanism of lily bulb dormancy release. Next, we will focus on the study of hormone signal transduction regulatory networks.

\section{Methods}

\section{Plant materials and treatments}

The lily bulbs used in this experiment were produced in Lanzhou, Gansu Province, China $\left(36^{\circ} 03^{\prime} \mathrm{N}\right.$, $103^{\circ} 40^{\prime} \mathrm{E}$ ), and harvested after the aboveground part of the plants withered in early October 2018 . The test materials were single-headed bulbs of uniform size (12-14 cm in circumference) and mass (30 $\pm 2 \mathrm{~g})$ that were free of diseases. Coconut bran with good structure and a water content of $70 \%-75 \%$ was used for cold storage of the bulbs at $4{ }^{\circ} \mathrm{C}$ for 120 days. The length of buds was measured every 10 days, and the germination rate was calculated after 15 days in the greenhouse. According to the germination rate and plant growth after low temperature treatment, the dormant bulbs at $100 \mathrm{~d}$ were determined to be released. Therefore, all the experiments in this study were carried out in three points: 0, 50 and $100 \mathrm{~d}$. The scales were removed, and the buds were collected. Fresh samples were immediately frozen in liquid nitrogen and stored at $-80^{\circ} \mathrm{C}$ for analysis. Each experiment was performed with three biological repeats.

\section{Determination of endogenous hormone contents}

\section{HPLC-MS/MS analysis of GAs}

A Qtrap6500 mass spectrometer was used for quantitative analysis of GAs using ESI-HPLC-MS/MS. In the three stages, approximately 1.0-1.6 g of fresh plant samples was weighed. After being ground in 
liquid nitrogen, $10 \mathrm{ml}$ acetonitrile solution was added to the plant samples, and the mixture was shaken at $4{ }^{\circ} \mathrm{C}$ for 8 hours and then centrifuged for $5 \mathrm{~min}$. The supernatant was taken and precipitated again, followed by addition of $10 \mathrm{~mL}$ of acetonitrile solution. In the absence of light, the organic phase was blow-dried with nitrogen, and the GA standard solution was prepared with $400 \mathrm{~L}$ methanol $(0.1 \%$ formic acid) as the solvent. The solution was filtered through $0.22 \mathrm{~m}$ membranes and detected by HPLC-MS/MS.

\section{HPLC-MS/MS analysis of IAA, ABA, SA, JA, MESA, and MEJA}

At the three sampling stages, $1-2 \mathrm{~g}$ of sample was weighed into a glass tube, and $10 \mathrm{ml}$ of isopropanol/hydrochloric acid extraction buffer was added to the powder, followed by shaking at $4^{\circ} \mathrm{C}$ for $30 \mathrm{~min}$. After the addition of $20 \mathrm{ml}$ of dichloromethane, the mixture was shaken again for $30 \mathrm{~min}$. Centrifugation was performed again for $5 \mathrm{~min}$, and the lower organic phase was collected. In the absence of light, the standard solutions of IAA, ABA, SA, JA, MESA and MEJA were prepared with $400 \mathrm{~L}$ methanol ( $0.1 \%$ formic acid) as the solvent. The solution was filtered through $0.22 \mathrm{~m}$ membranes and detected by HPLC-MS/MS.

\section{Illumina sequencing, differential gene expression analysis, gene functional annotation}

The RNA of lily samples was extracted by the CTAB method, and then the RNA purity (OD260/280 and OD260/230) and integrity were determined by a Nano Photometer and Agilent 2100 Bio-Analyzer, respectively. After evaluation, the library was constructed for transcriptome sequencing, and Qubit2.0 was used for preliminary quantification. After ensuring that the insert size detection was in line with expectations, qRT-PCR was used for accurate quantification of the effective concentration of the library. Illumina HiSeq sequencing was performed after pooling different libraries according to the effective concentration and target data volume requirements. Raw reads were obtained from sequencing, and reads with adaptors and low-quality reads were filtered out. Then, Trinity was used to splice the clean reads. The transcriptome obtained by Trinity splicing was used as a reference sequence for RSEM, and the clean reads of each sample were mapped on reference. The results were statistically compared to obtain the read count number of each sample, and FPKM conversion was performed to analyze the gene expression levels. The DEGs were analyzed by DESeq265, and the screening threshold was p. adj. $<0.05$ and |log2FoldChange|>1. In the process of difference analysis, the p-values obtained from the original hypothesis test were corrected. TF prediction was performed by iTAK software.

In order to obtain more comprehensive gene function annotation information, seven databases were employed for gene functional annotation, including the KEGG, GO, Nr, Pfam, Nt, KOG/COG, and Swiss-Prot databases. Through comparative annotation with the $\mathrm{Nr}$ library, the similarity between the gene sequence of this species and the gene sequences of related species as well as the functional information of the genes of this species were obtained. After the genes were annotated with GO, the successfully annotated genes were classified according to the next level of the three major $\mathrm{GO}$ categories (BP biological process, CC cellular component, and MF molecular function). Compared with the cluster of Orthologous Groups of Proteins database, the possible protein functions of unigenes were predicted and classified. After KO 
annotation, the genes were classified according to the KEGG metabolic pathways in which they participated, and the relevant metabolic pathways were annotated.

\section{Correlation analysis}

For the correlation analysis between hormone contents and the transcriptome, the average contents for each endogenous hormone obtained with all biological replicates and the average DEG expression levels in the transcriptome data were obtained, and the correlation was calculated using the "Spearman" algorithm. Heat maps were used to show the link between genes and metabolites.

\section{QRT PCR verification}

Twenty DEGs were randomly selected, and primers were designed using Primer Premier 5.0 for real-time quantitative PCR detection. After starting at $95^{\circ} \mathrm{C}$ for $30 \mathrm{sec}$, the fusion curve was analyzed after 40 cycles of $95^{\circ} \mathrm{C}$ for $30 \mathrm{sec}, 60^{\circ} \mathrm{C}$ for $10 \mathrm{sec}$, and $72^{\circ} \mathrm{C}$ for $30 \mathrm{sec}$. Quantitative results were analyzed using the $2^{\triangle \triangle C T}$ method. Three technical replicates and three biological replicates were performed for each group.

\section{Abbreviations}

DEGs: differentially expressed genes; TF: transcript factor; DD: deep dormancy; MD: middle dormancy; DR: Dormancy release.

\section{Declarations}

\section{Acknowledgements}

Not applicable

\section{Funding}

This work was financed by the national key research and development project "Post-harvest quality maintenance mechanism and regulation of key flowers" (2018YFD1000407, Ministry of Science and Technology of the People's Republic of China).

\section{Author contributions}

$X Y F, Y Y$ and HMS conceived and designed the experiments. ML, LLF and YQZ were involved in the sampling and preliminary processing of the tests. CXW and TYH assisted the authors in data collation and analysis. All of the authors read and approved the final manuscript.

\section{Ethics approval and consent to participate}

Not applicable 


\section{Consent for publication}

Not applicable

\section{Competing of interest}

The authors declare no conflicts of interest in the submission of this manuscript.

\section{Availability of data and material}

The datasets used and/or analysed during the current study available from the corresponding author on reasonable request.

\section{References}

1. Shu K, Meng YJ, Shuai HW, Liu WG, Du JB, Liu J, Yang WY. Dormancy and germination: How does the crop seed decide? Plant Biol. 2015;17(6):1104-12.

2. Wang R, Wang G, Zhao Q, Zhang Y, An L, Wang Y. Expression, purification and characterization of the Lily symptomless virus coat protein from Lanzhou Isolate. Virol J. 2010;7:34.

3. Wang W, Su XX, Tian ZP, Liu Y, Zhou YW, He M. Transcriptome profiling provides insights into dormancy release during cold storage of Lilium pumilum. Bmc Genomics 2018, 19.

4. Richardson AC, Walton EF, Meekings JS, Boldingh HL. Carbohydrate changes in kiwifruit buds during the onset and release from dormancy. Sci Hortic-Amsterdam. 2010;124(4):463-8.

5. Fadon E, Herrero M, Rodrigo J. Dormant Flower Buds Actively Accumulate Starch over Winter in Sweet Cherry. Frontiers in plant science. 2018;9:171.

6. Zhang Z, Zhuo X, Zhao K, Zheng T, Han Y, Yuan C, Zhang Q. Transcriptome Profiles Reveal the Crucial Roles of Hormone and Sugar in the Bud Dormancy of Prunus mume. Scientific reports. 2018;8(1):5090.

7. Rinne PL, Welling A, Vahala J, Ripel L, Ruonala R, Kangasjarvi J, van der Schoot C. Chilling of dormant buds hyperinduces FLOWERING LOCUS T and recruits GA-inducible 1,3-beta-glucanases to reopen signal conduits and release dormancy in Populus. Plant Cell. 2011;23(1):130-46.

8. Sonnewald S, Sonnewald U. Regulation of potato tuber sprouting. Planta. 2014;239(1):27-38.

9. Subbaraj AK, Funnell KA, Woolley DJ. Dormancy and Flowering Are Regulated by the Reciprocal Interaction Between Cytokinin and Gibberellin in Zantedeschia. J Plant Growth Regul. 2010;29(4):487-99.

10. Wu J, Seng SS, Sui JJ, Vonapartis E, Luo X, Gong BH, Liu C, Wu CY, Liu C, Zhang FQ, et al: Gladiolus hybridus ABSCISIC ACID INSENSITIVE 5 (GhABI5) is an important transcription factor in ABA signaling that can enhance Gladiolus corm dormancy and Arabidopsis seed dormancy. Frontiers in plant science 2015, 6. 
11. Thalmann M, Pazmino D, Seung D, Horrer D, Nigro A, Meier T, Kolling K, Pfeifhofer HW, Zeeman SC, Santelia D. Regulation of Leaf Starch Degradation by Abscisic Acid Is Important for Osmotic Stress Tolerance in Plants. Plant Cell. 2016;28(8):1860-78.

12. Rubio S, Noriega X, Perez FJ. ABA promotes starch synthesis and storage metabolism in dormant grapevine buds. Journal of plant physiology. 2019;234-235:1-8.

13. Zhong CM, Xu H, Ye ST, Wang SY, Li LF, Zhang SC, Wang XJ. Gibberellic Acid-Stimulated Arabidopsis6 Serves as an Integrator of Gibberellin, Abscisic Acid, and Glucose Signaling during Seed Germination in Arabidopsis(1[OPEN]). Plant Physiol. 2015;169(3):2288-303.

14. Xie Z, Zhang ZL, Zou X, Yang G, Komatsu S, Shen QJ. Interactions of two abscisic-acid induced WRKY genes in repressing gibberellin signaling in aleurone cells. The Plant journal: for cell molecular biology. 2006;46(2):231-42.

15. Niu Q, Li J, Cai D, Qian M, Jia H, Bai S, Hussain S, Liu G, Teng Y, Zheng X. Dormancy-associated MADS-box genes and microRNAs jointly control dormancy transition in pear (Pyrus pyrifolia white pear group) flower bud. J Exp Bot. 2016;67(1):239-57.

16. Resentini F, Felipo-Benavent A, Colombo L, Blazquez MA, Alabadi D, Masiero S. TCP14 and TCP15 mediate the promotion of seed germination by gibberellins in Arabidopsis thaliana. Molecular plant. 2015;8(3):482-5.

17. Yang Q, Yang B, Li J, Wang Y, Tao R, Yang F, Wu X, Yan X, Ahmad M, Shen J, et al. ABA-responsive ABRE-BINDING FACTOR3 activates DAM3 expression to promote bud dormancy in Asian pear. Plant Cell Environment. 2020;43(6):1360-75.

18. Dong Y, Guan M, Wang L, Yuan L, Sun X, Liu S. Transcriptome Analysis of Low-Temperature-Induced Breaking of Garlic Aeria/ Bulb Dormancy. International journal of genomics. 2019;2019:9140572.

19. Wang Y, Liu X, Su H, Yin S, Han C, Hao D, Dong $X$. The regulatory mechanism of chilling-induced dormancy transition from endo-dormancy to non-dormancy in Polygonatum kingianum Coll.et Hems/ rhizome bud. Plant molecular biology. 2019;99(3):205-17.

20. Xu PL, Tang GY, Cui WP, Chen GX, Ma CL, Zhu JQ, Li PX, Shan L, Liu ZJ, Wan SB. Transcriptional Differences in Peanut (Arachis hypogaea L.) Seeds at the Freshly Harvested, After-ripening and Newly Germinated Seed Stages: Insights into the Regulatory Networks of Seed Dormancy Release and Germination. Plos One 2020, 15(1).

21. Vimont N, Fouche M, Campoy JA, Tong M, Arkoun M, Yvin JC, Wigge PA, Dirlewanger E, Cortijo S, Wenden B. From bud formation to flowering: transcriptomic state defines the cherry developmental phases of sweet cherry bud dormancy. Bmc Genomics. 2019;20(1):974.

22. Hooykass PJJ, Hall MA. K.R L: Biochemistry and molecular biology of plant hormones. Elservier Amsterdam. 1999;75:1418-8.

23. Kim J, Lee JG, Hong Y, Lee EJ. Analysis of eight phytohormone concentrations, expression levels of ABA biosynthesis genes, and ripening-related transcription factors during fruit development in strawberry. Journal of plant physiology. 2019;239:52-60. 
24. Naz RMM, Li M, Ramzan S, Li G, Liu J, Cai X, Xie C. QTL mapping for microtuber dormancy and $\mathrm{GA}_{3}$ content in a diploid potato population. Biology open 2018, 7(1).

25. Suh JK, Kwack BH. Physiological changes in the course of bulbing and dormancy-breaking of gladiolus (Gladiolus gandavensis L.). Journal of the Korean Society for Horticultural ence 1992.

26. Wei YZH, Meng X. Effects of GA_3 and 6-BA Treatments on Changes of Three Endogenous Phytohormones Contents in Tulip Bulbs. Plant Physiology Journal. 2013;49:161-6.

27. Lian QL, Xin HB, Li XX, Zhong XH, Yin YL, Yi MF. Isolation, characterization and expression analysis of the genes-GhAOS, GhAOC and GhOPR3: Encoding the key enzymes involved in jasmonic acid biosynthesis in Gladiolus hybridus. Sci Hortic-Amsterdam. 2013;154:88-95.

28. Smeekens S, Hellmann HA. Sugar sensing and signaling in plants. Frontiers in plant science. 2014;5:113.

29. Son SH, Chitnis VR, Liu A, Gao F, Nguyen TN, Ayele BT. Abscisic acid metabolic genes of wheat (Triticum aestivum L.): identification and insights into their functionality in seed dormancy and dehydration tolerance. Planta. 2016;244(2):429-47.

30. Destefano-Beltran L, Knauber D, Huckle L, Suttle JC. Effects of postharvest storage and dormancy status on ABA content, metabolism, and expression of genes involved in ABA biosynthesis and metabolism in potato tuber tissues. Plant molecular biology. 2006;61(4-5):687-97.

31. Rubinovich L, Weiss $D$. The Arabidopsis cysteine-rich protein GASA4 promotes GA responses and exhibits redox activity in bacteria and in planta. Plant J. 2010;64(6):1018-27.

32. Zeng DE, Hou P, Xiao FM, Liu YS. Overexpression of Arabidopsis XERICO gene confers enhanced drought and salt stress tolerance in rice (Oryza Sativa L.). J Plant Biochem Biot. 2015;24(1):56-64.

33. Footitt S, Douterelo-Soler I, Clay H, Finch-Savage WE. Dormancy cycling in Arabidopsis seeds is controlled by seasonally distinct hormone-signaling pathways. P Natl Acad Sci USA. 2011;108(50):20236-41.

34. Shu K, Zhang H, Wang S, Chen M, Wu Y, Tang S, Liu C, Feng Y, Cao X, Xie Q. ABI4 regulates primary seed dormancy by regulating the biogenesis of abscisic acid and gibberellins in Arabidopsis. PLoS Genet. 2013;9(6):e1003577.

35. Cantoro R, Crocco CD, Benech-Arnold RL, Rodriguez MV. In vitro binding of Sorghum bicolor transcription factors $A B I 4$ and $A B I 5$ to a conserved region of a GA 2-OXIDASE promoter: possible role of this interaction in the expression of seed dormancy. J Exp Bot. 2013;64(18):5721-35.

36. Kanai $M$, Nishimura $M$, Hayashi $M$ : A peroxisomal $A B C$ transporter promotes seed germination by inducing pectin degradation under the control of ABI5. The Plant journal: for cell and molecular biology 2010, 62(6):936-947.

37. Liu X, Hou X. Antagonistic Regulation of ABA and GA in Metabolism and Signaling Pathways. Frontiers in plant science. 2018;9:251.

38. Liu X, Hu P, Huang M, Tang Y, Li Y, Li L, Hou X. The NF-YC-RGL2 module integrates GA and ABA signalling to regulate seed germination in Arabidopsis. Nature communications. 2016;7:12768. 
39. Jiang W, Yu D. Arabidopsis WRKY2 transcription factor mediates seed germination and postgermination arrest of development by abscisic acid. BMC Plant Biol. 2009;9:96-6.

40. Yang B, Song Z, Li C, Jiang J, Zhou Y, Wang R, Wang Q, Ni C, Liang Q, Chen H, et al. RSM1, an Arabidopsis MYB protein, interacts with $\mathrm{HY} 5 / \mathrm{HYH}$ to modulate seed germination and seedling development in response to abscisic acid and salinity. PLoS Genet. 2018;14(12):e1007839.

41. Xing L, Zhao Y, Gao J, Xiang C, Zhu JK. The ABA receptor PYL9 together with PYL8 plays an important role in regulating lateral root growth. Scientific reports. 2016;6:27177.

42. Wu J, Jin Y, Liu C, Vonapartis E, Liang J, Wu W, Gazzarrini S, He J, Yi M. GhNAC83 inhibits corm dormancy release by regulating ABA signaling and cytokinin biosynthesis in Gladiolus hybridus. J Exp Bot. 2019;70(4):1221-37.

43. Wu R, Tomes S, Karunairetnam S, Tustin SD, Hellens RP, Allan AC, Macknight RC, Varkonyi-Gasic E. SVP-like MADS Box Genes Control Dormancy and Budbreak in Apple. Frontiers in plant science. 2017;8:477.

44. Y K, T T, H Y. R T: Simultaneous down-regulation of DORMANCY-ASSOCIATEDMADS-box 6and SOC1during dormancy release in Japanese apricot (Prunus mume) flower buds. Horticulture Science Biotech. 2016;91:476-82.

45. Lee J, Lee I. Regulation and function of SOC1, a flowering pathway integrator. J Exp Bot. 2010;61(9):2247-54.

46. Wang Q, Xu G, Zhao X, Zhang Z, Wang X, Liu X, Xiao W, Fu X, Chen X, Gao D, et al. Transcription factor TCP20 regulates peach bud endodormancy by inhibiting DAM5/DAM6 and interacting with ABF2. J Exp Bot. 2020;71(4):1585-97.

47. Wu J, Wu W, Liang J, Jin Y, Gazzarrini S, He J, Yi M. GhTCP19 Transcription Factor Regulates Corm Dormancy Release by Repressing GhNCED Expression in Gladiolus. Plant Cell Physiol. 2019;60(1):52-62.

48. Hu Y, Han X, Yang M, Zhang M, Pan J, Yu D. The Transcription Factor INDUCER OF CBF EXPRESSION1 Interacts with ABSCISIC ACID INSENSITIVE5 and DELLA Proteins to Fine-Tune Abscisic Acid Signaling during Seed Germination in Arabidopsis. Plant Cell. 2019;31(7):1520-38.

\section{Tables}

Table.1 Differential TFs during dormancy release in Lilium davidivar. unicolor 


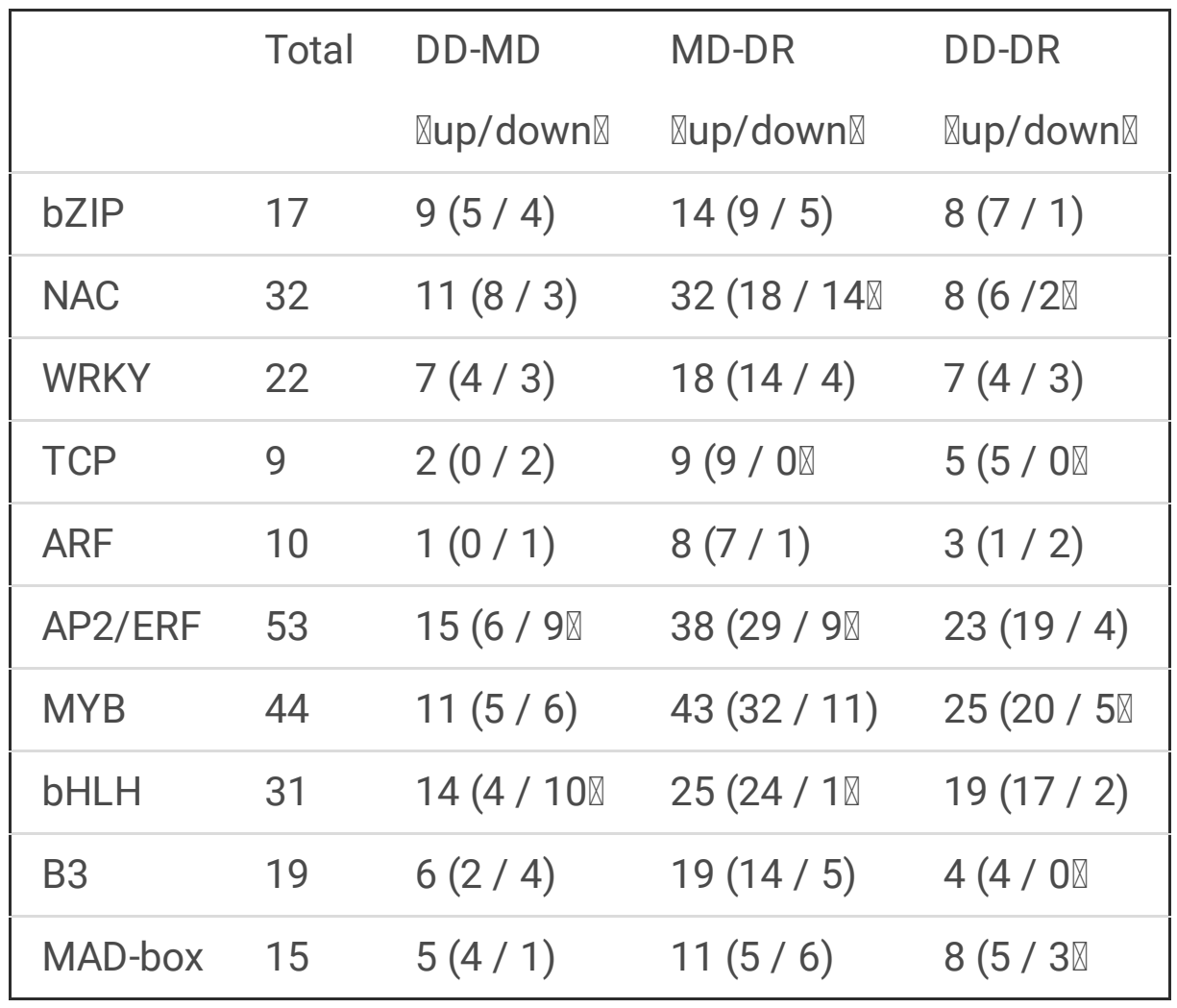

\section{Figures}
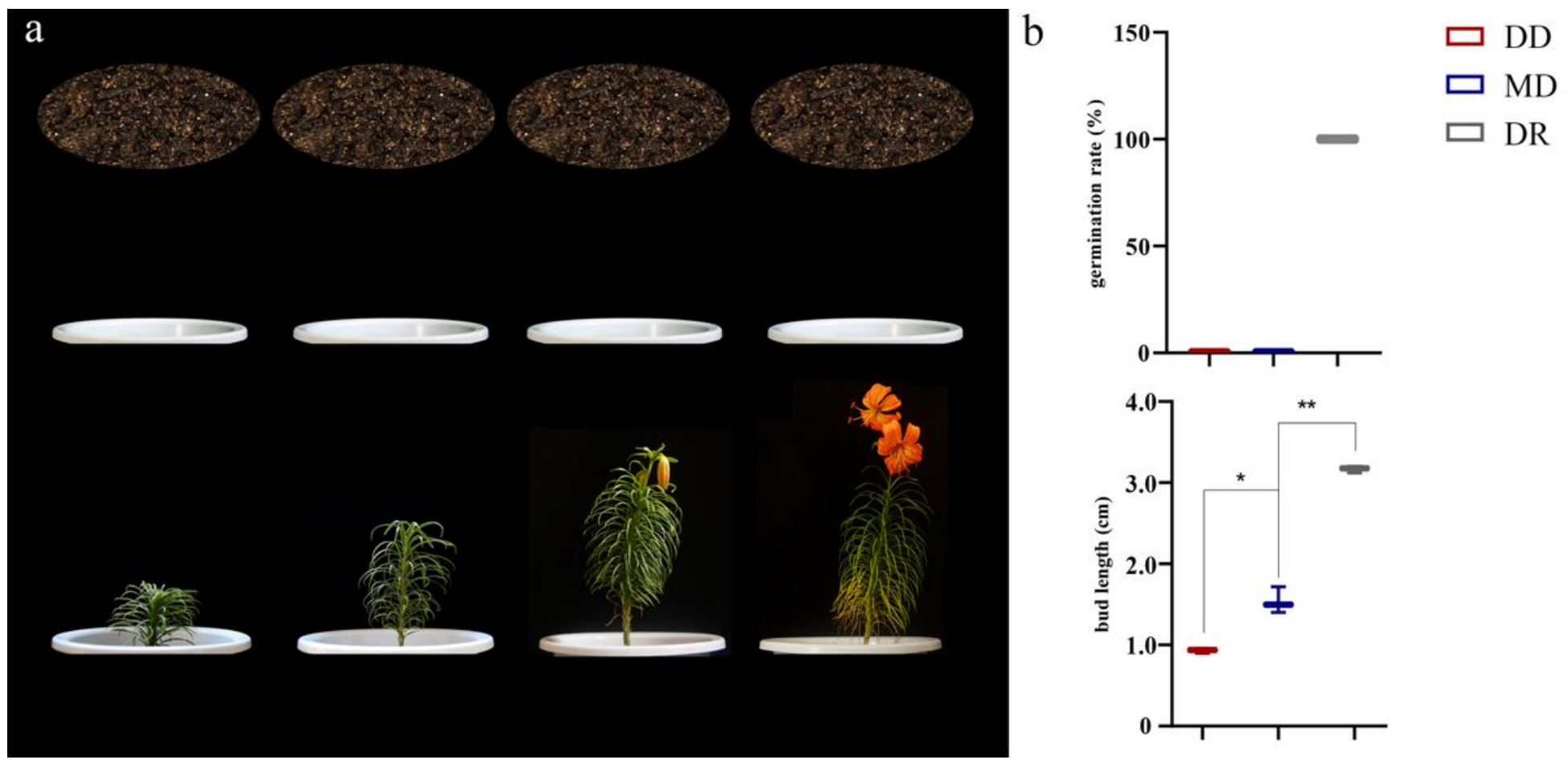

Figure 1 
Images illustrating the the difference of lily bulb growth characters in DD, MD and DR states a. The growth of lily bulbs at $1 \mathrm{~m}, 2 \mathrm{~m}, 3 \mathrm{~m}$ and $4 \mathrm{~m}$ after potted under DDIMD and DR stage. b. Growth trait comparison of Lily bulbs under three stages. "**” indicated the significant difference between the two sets of data $(\mathrm{P} \otimes 0.01)$.
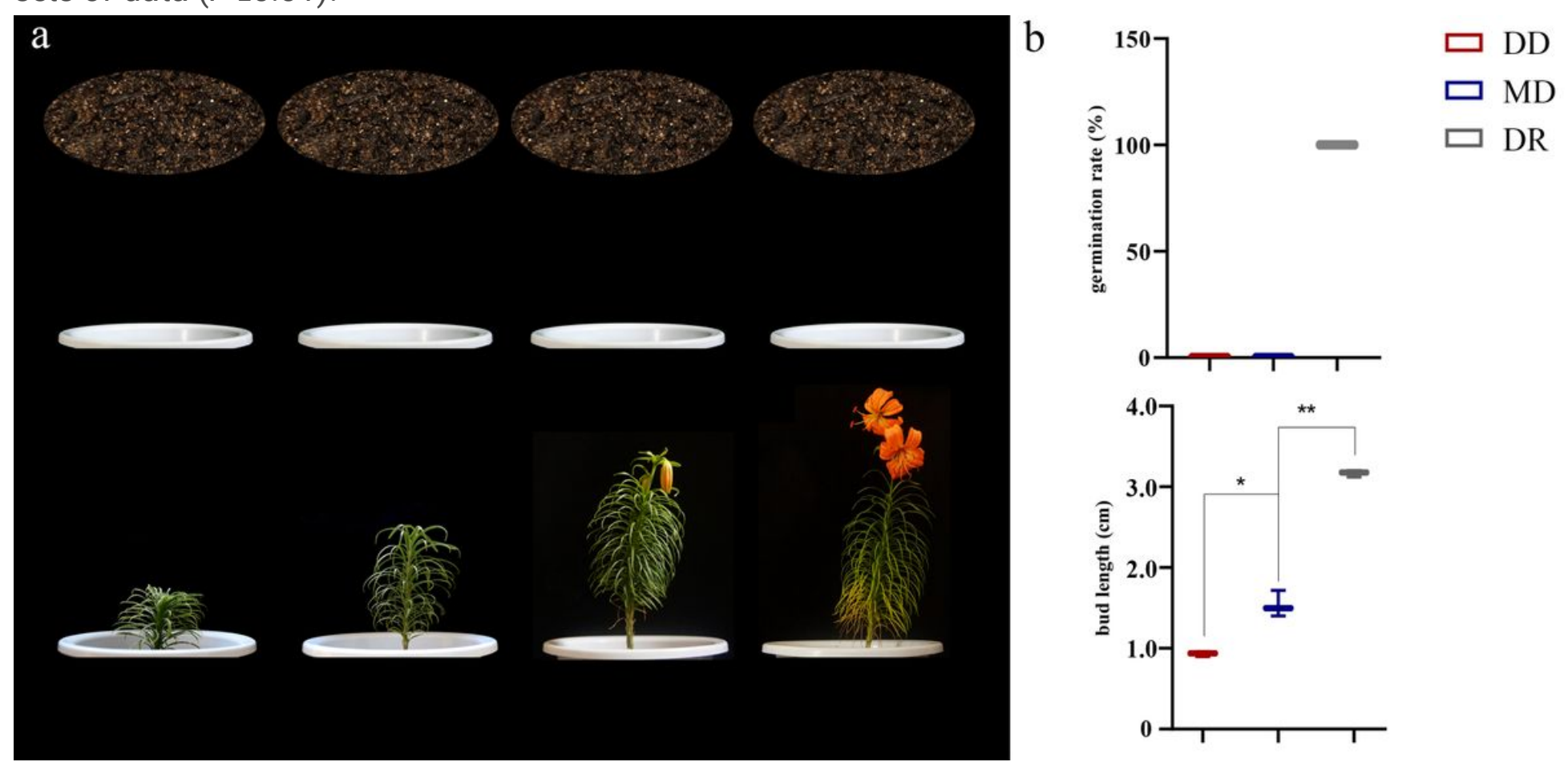

\section{Figure 1}

Images illustrating the the difference of lily bulb growth characters in DD, MD and DR states a. The growth of lily bulbs at $1 \mathrm{~m}, 2 \mathrm{~m}, 3 \mathrm{~m}$ and $4 \mathrm{~m}$ after potted under DDIMD and DR stage. b. Growth trait comparison of Lily bulbs under three stages. "**” indicated the significant difference between the two sets of data $(P \otimes 0.01)$. 

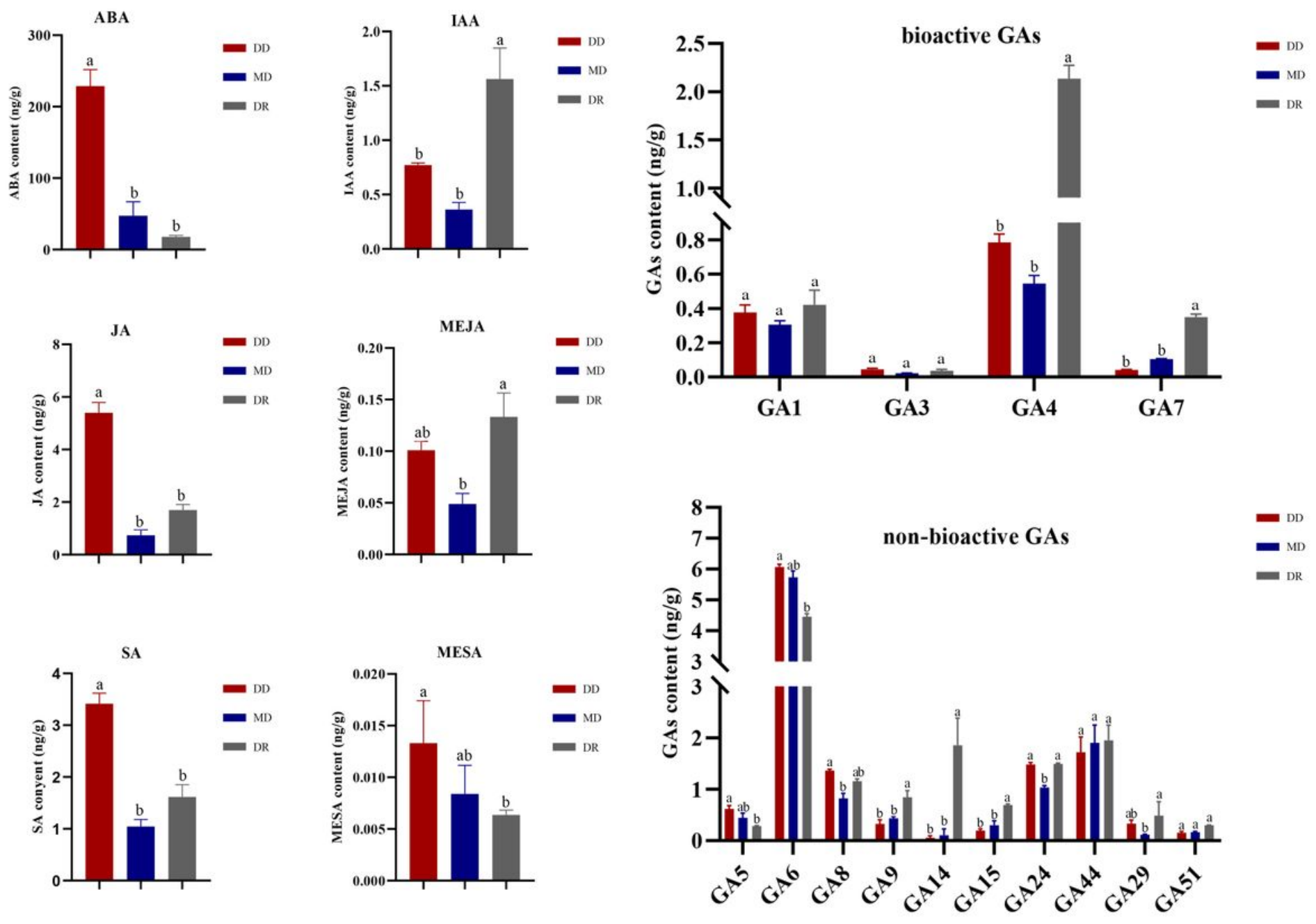

Figure 2

Changes of endogenous hormone content in bulb of Lily during dormancy release at low temperature. 

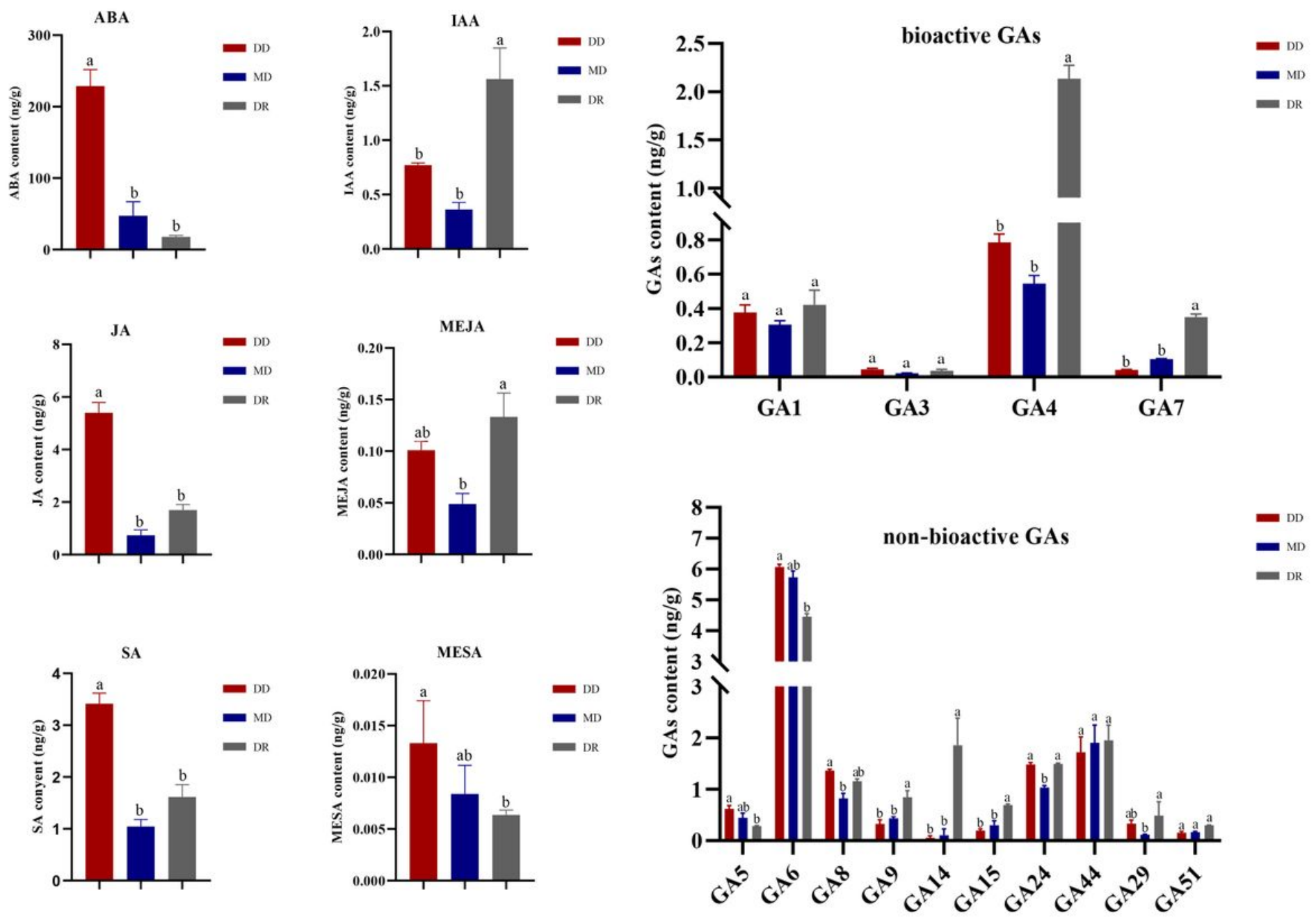

Figure 2

Changes of endogenous hormone content in bulb of Lily during dormancy release at low temperature. 

- Glycolysis / Gluconeogenesis
= Citrate cycle (TCA cycle)
- Pentose phosphate pathway
= Fructose and mannose metabolism
= Amino sugar and nucleotide sugar metabolism
- Pentose and glucuronate interconversions
= Galactose metabolism
- Starch and sucrose metabolism
= Pyruvate metabolism
= Propanoate metabolism
- C5-Branched dibasic acid metabolism
- Ascorbate and aldarate metabolism
= Glyoxylate and dicarboxylate metabolism
= Butanoate metabolism
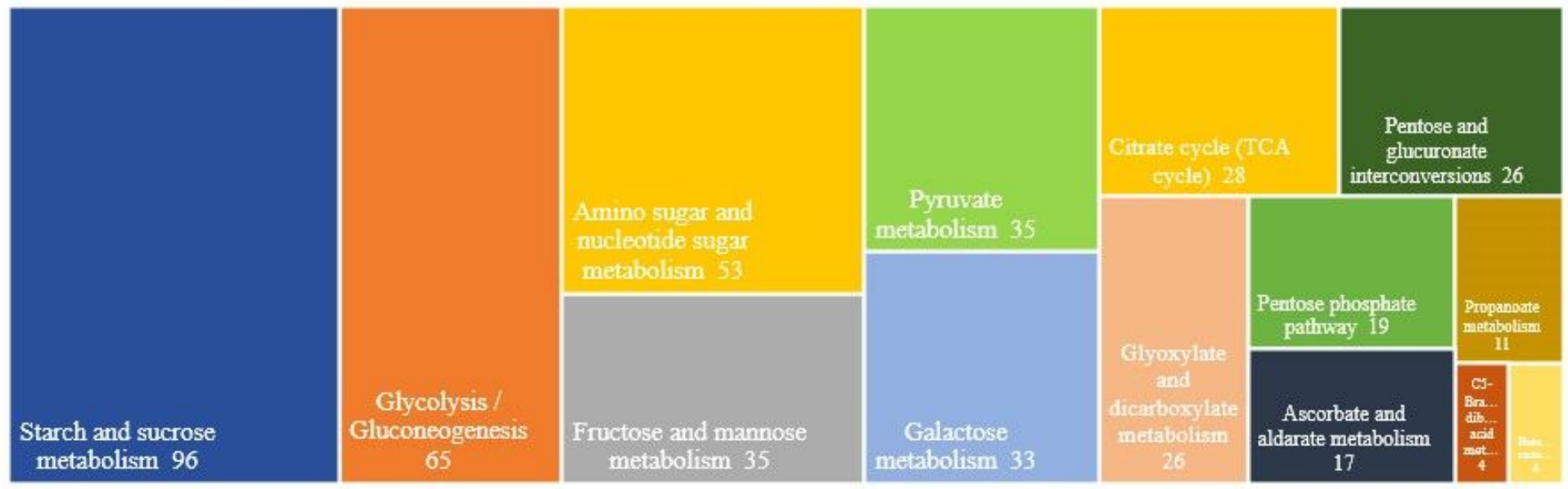

\section{Figure 3}

The number of DEGs enriched in carbohydrate metabolism related pathways.

= Glycolysis / Gluconeogenesis

= Pentose phosphate pathway

= Amino sugar and nucleotide sugar metabolism

= Galactose metabolism

= Pyruvate metabolism

- C5-Branched dibasic acid metabolism

= Glyoxylate and dicarboxylate metabolism
= Citrate cycle (TCA cycle)

= Fructose and mannose metabolism

- Pentose and glucuronate interconversions

- Starch and sucrose metabolism

- Propanoate metabolism

- Ascorbate and aldarate metabolism

= Butanoate metabolism
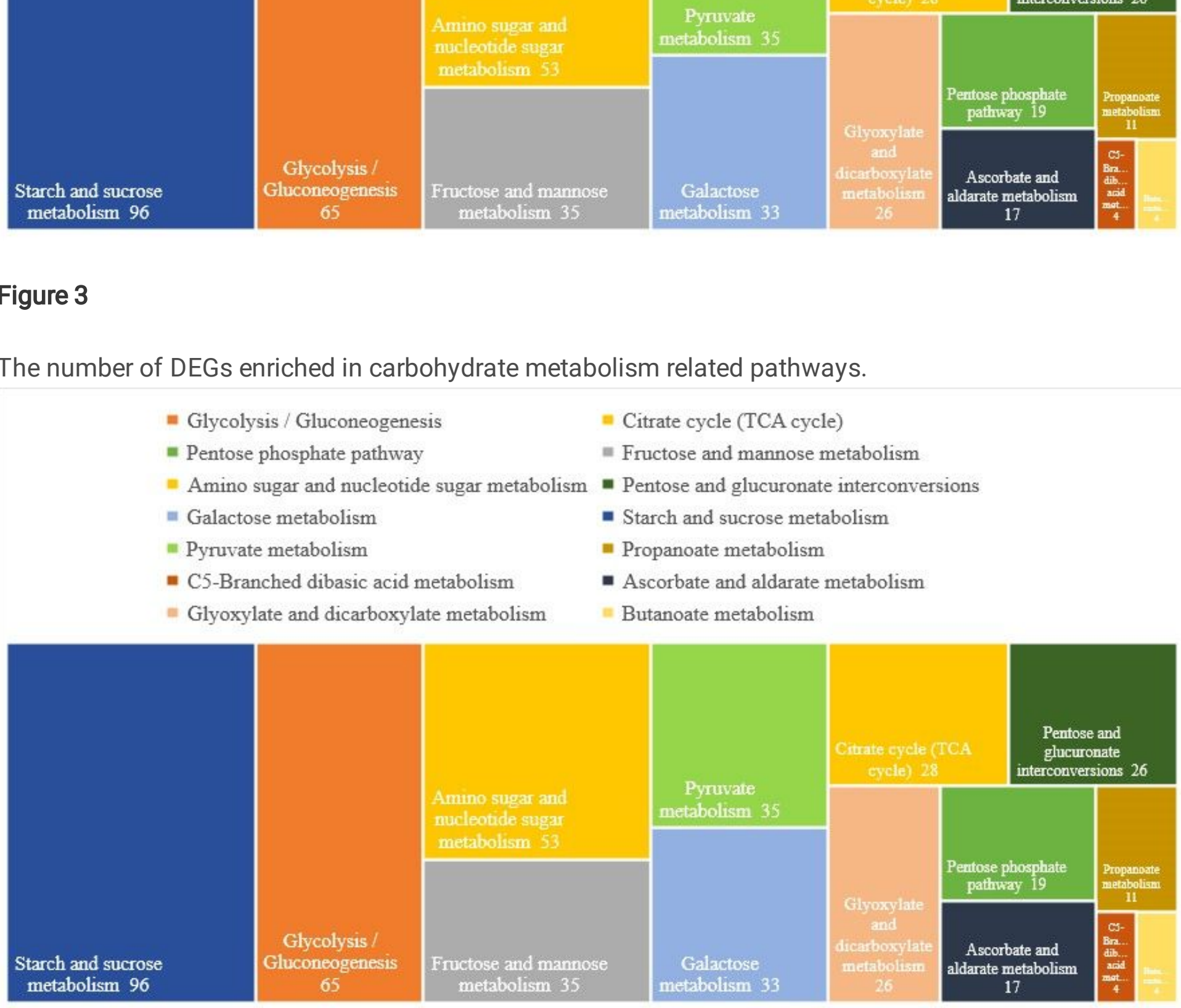

\section{Figure 3}

The number of DEGs enriched in carbohydrate metabolism related pathways. 
Glycolysis / Gluconeogenesis

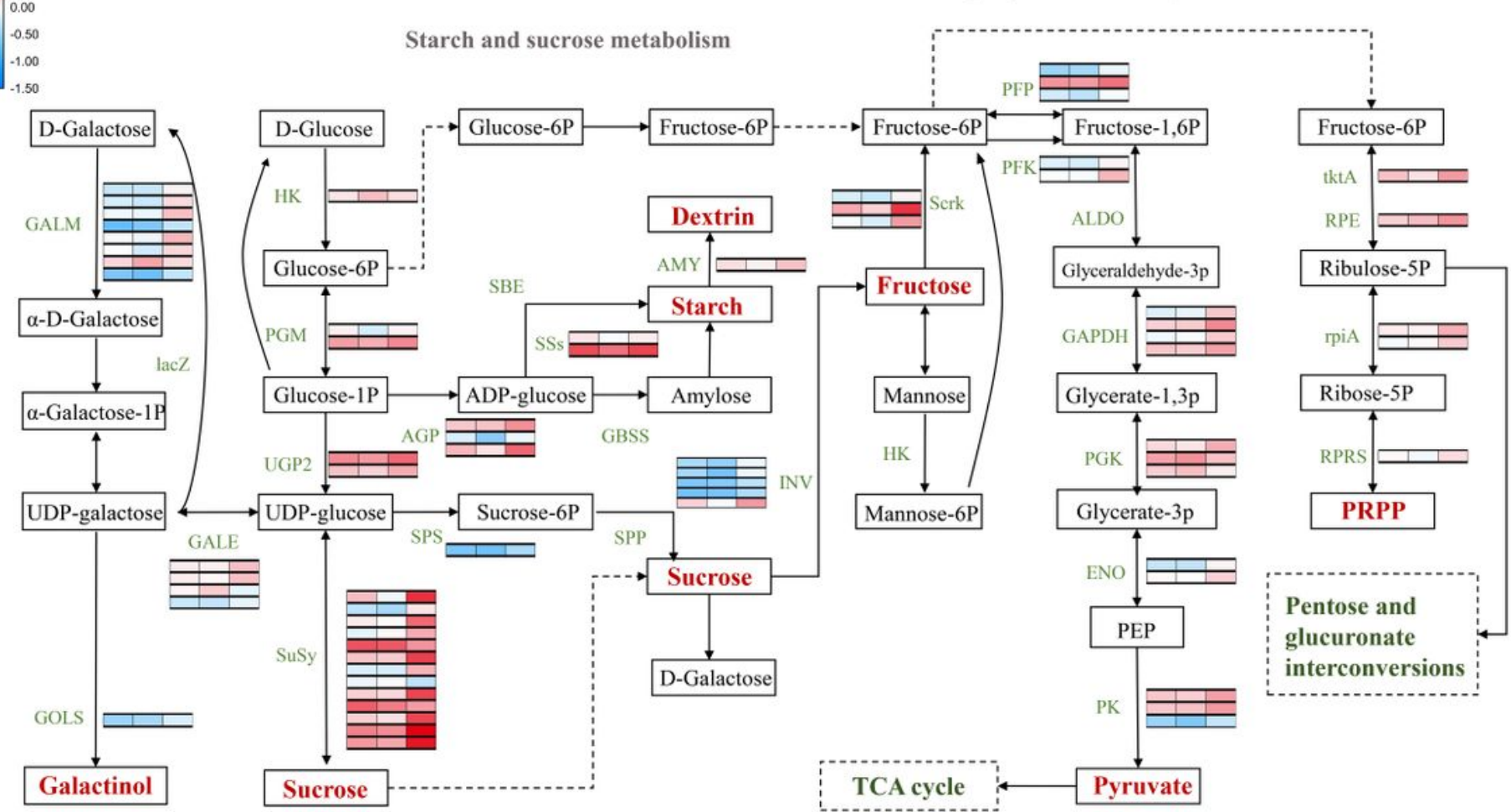

Galactose metabolism

Fructose and mannose metabolism

\section{Figure 4}

Heat map of DEGs involved in carbohydrate metabolism related pathways. The change of the color scale in heat map fro $\mathrm{m}$ blue to red represents indicated that the gene is up regulate, or down regulate. 
Glycolysis / Gluconeogenesis

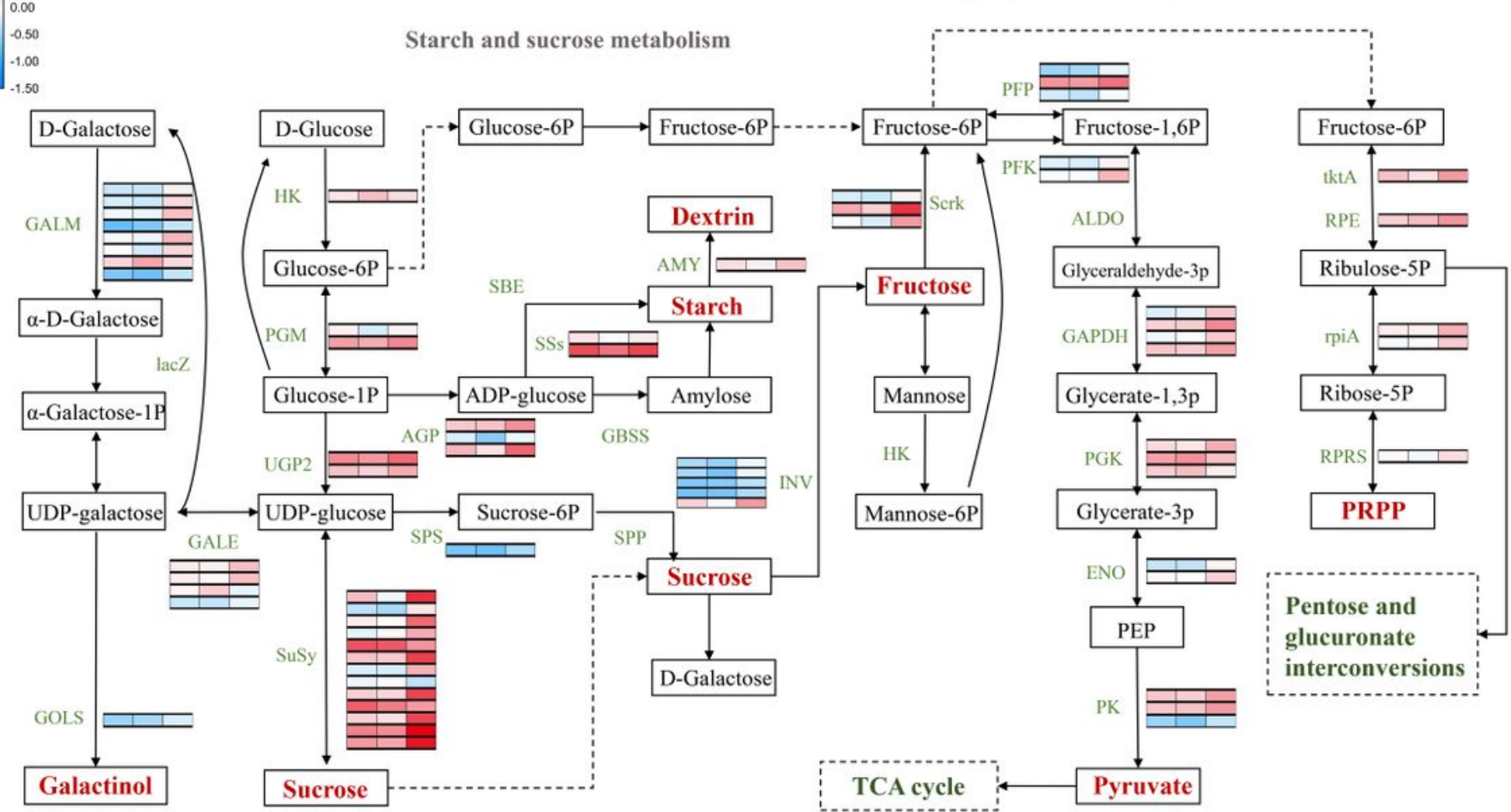

Galactose metabolism

Fructose and mannose metabolism

\section{Figure 4}

Heat map of DEGs involved in carbohydrate metabolism related pathways. The change of the color scale in heat map fro $\mathrm{m}$ blue to red represents indicated that the gene is up regulate, or down regulate. 
A

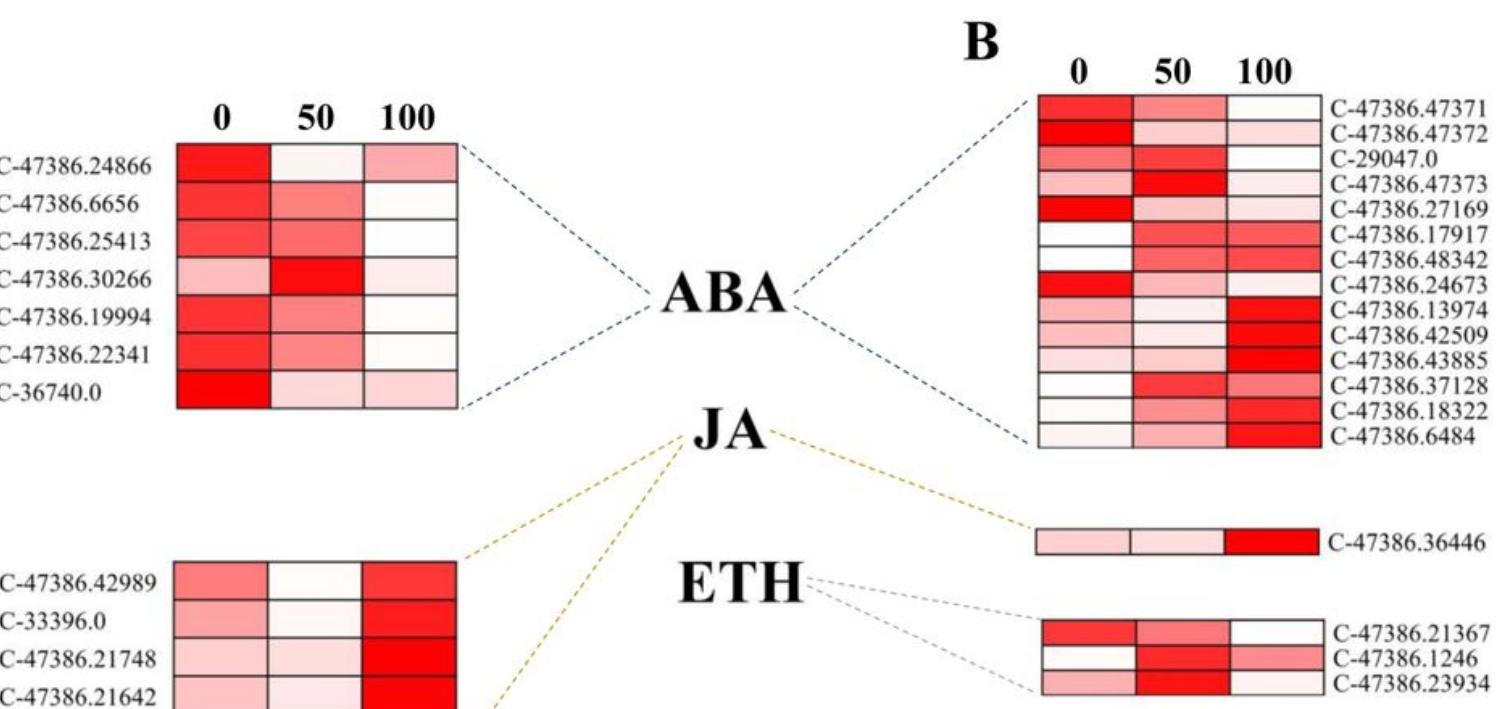

C-47386.44259
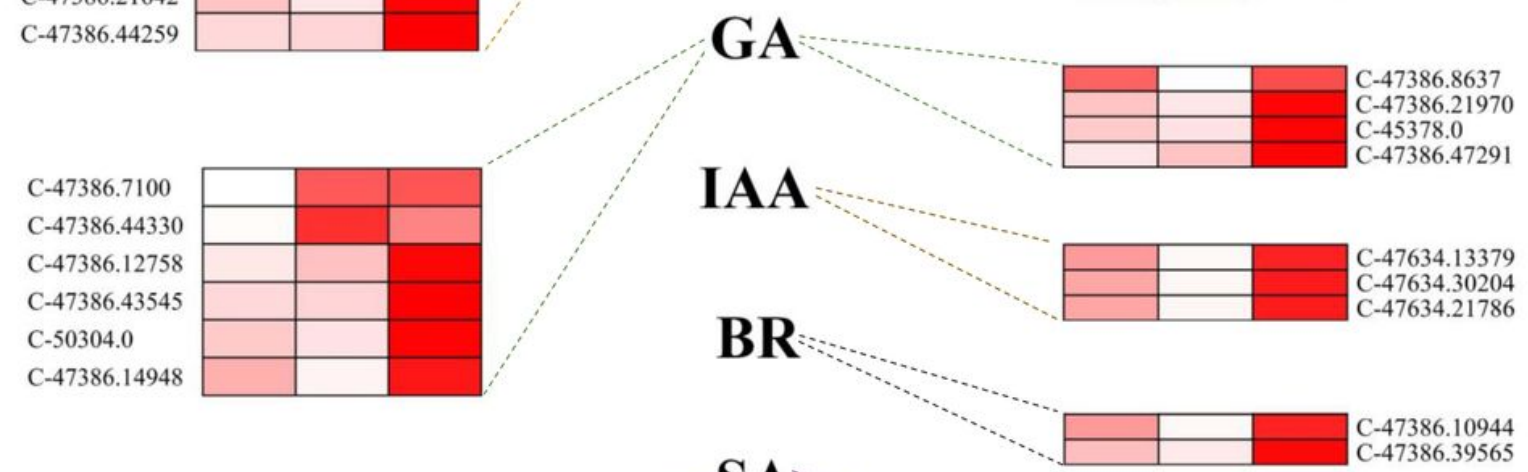

C-47386.38306

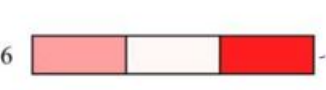

$\mathbf{S A}$

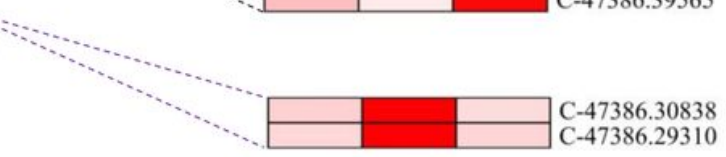

\section{Figure 5}

Heat map of major DEGs involved in metabolic and signal transduction pathway. A. Hormone metabolic pathway. B. Hormone signal transduction pathway 
A

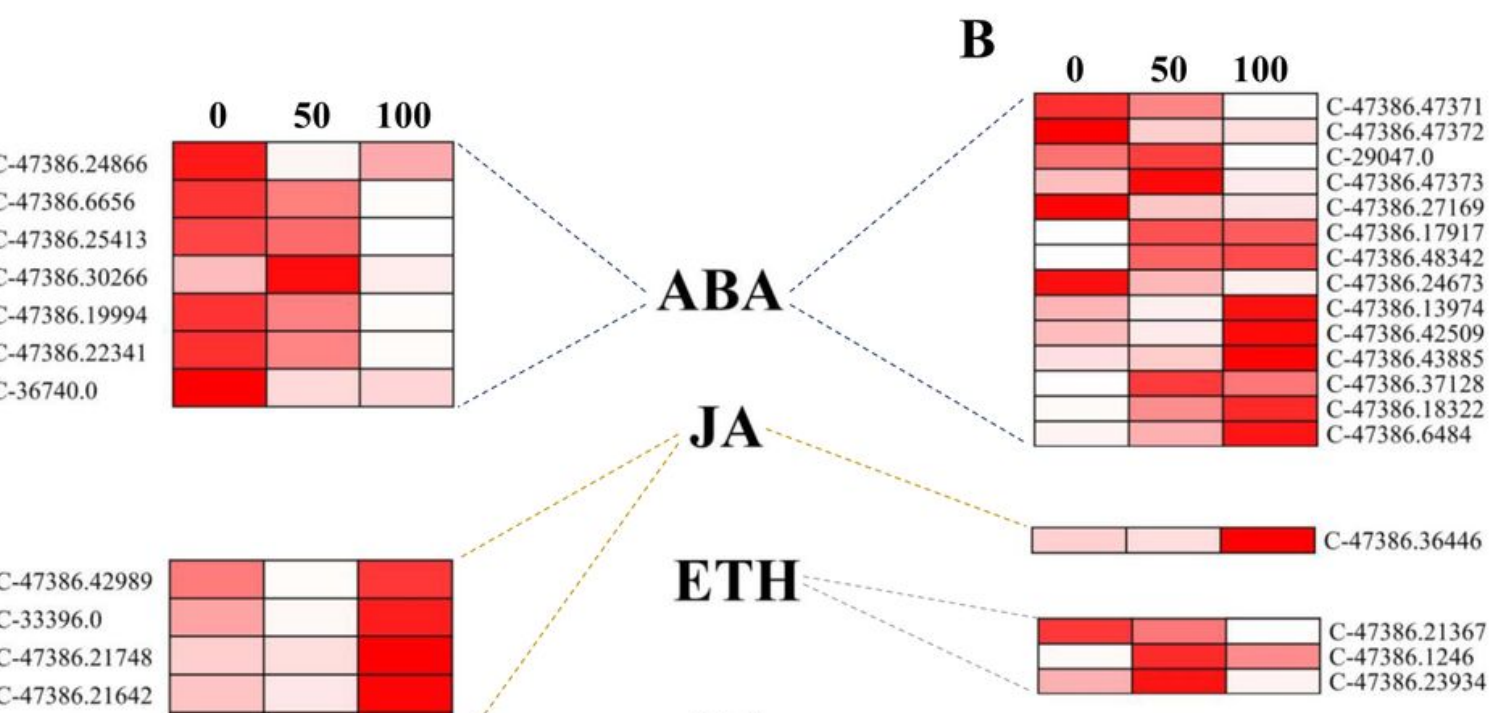

C- 47386.44259
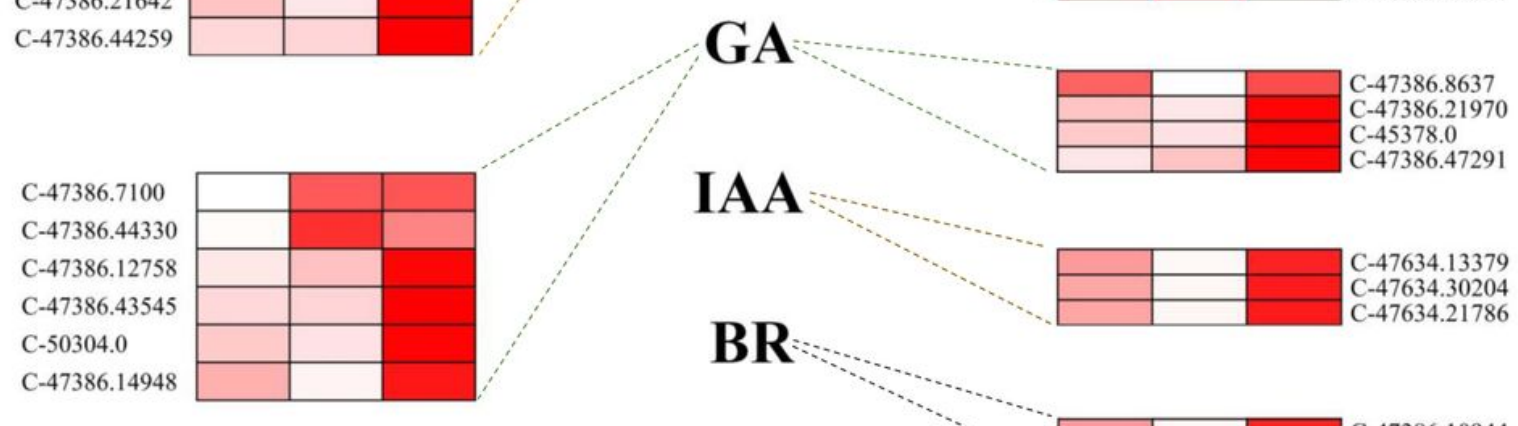

C-47386.7100

C-47386.44330

C-47386.1275

SA

C-47386.38306
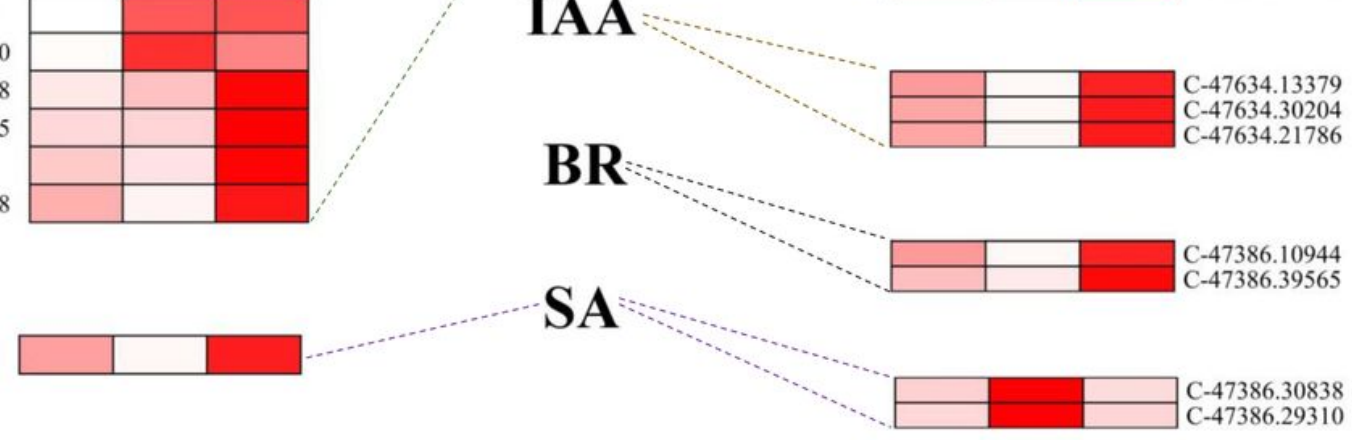

\section{Figure 5}

Heat map of major DEGs involved in metabolic and signal transduction pathway. A. Hormone metabolic pathway. B. Hormone signal transduction pathway 


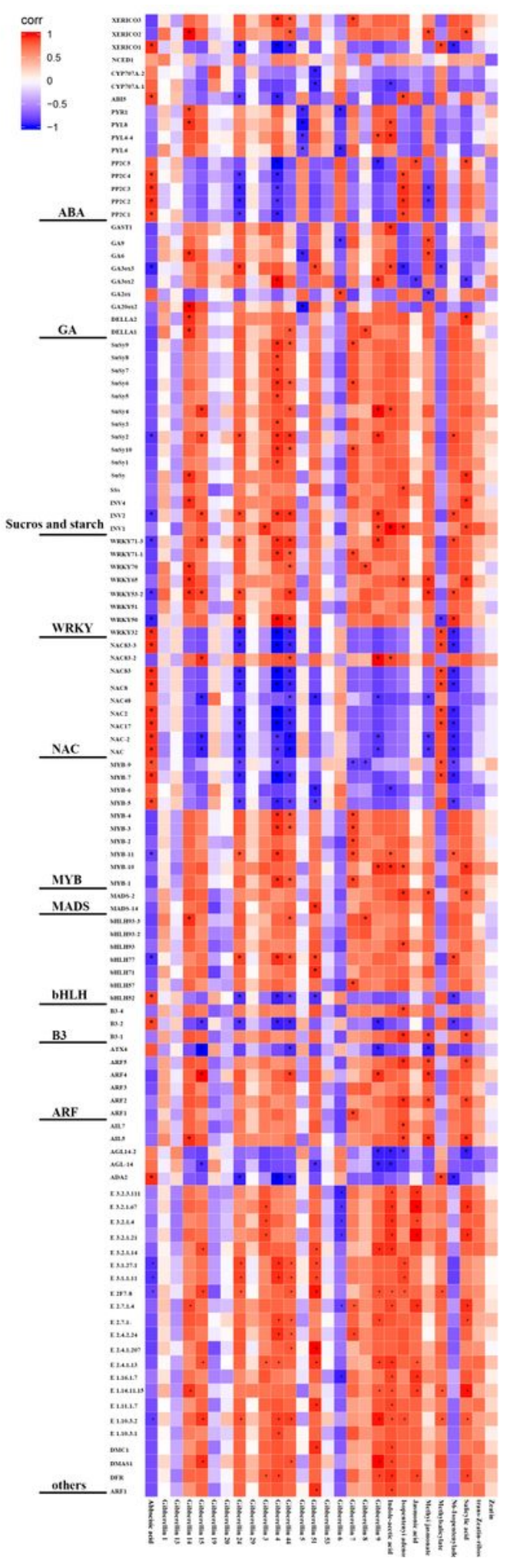

\section{Figure 6}

Heat map of the correlations between transcriptome and the targeted metabolome. The ordinate is the DEGs in the transcriptome, and the abscissa is the type of targeted metabolites. The red and blue represented the positive or negative correlation respectively. The * indicated that there is a significant correlation between genes and targeted metabolites ( $\mathrm{P} \mathrm{b} 0.05)$. 


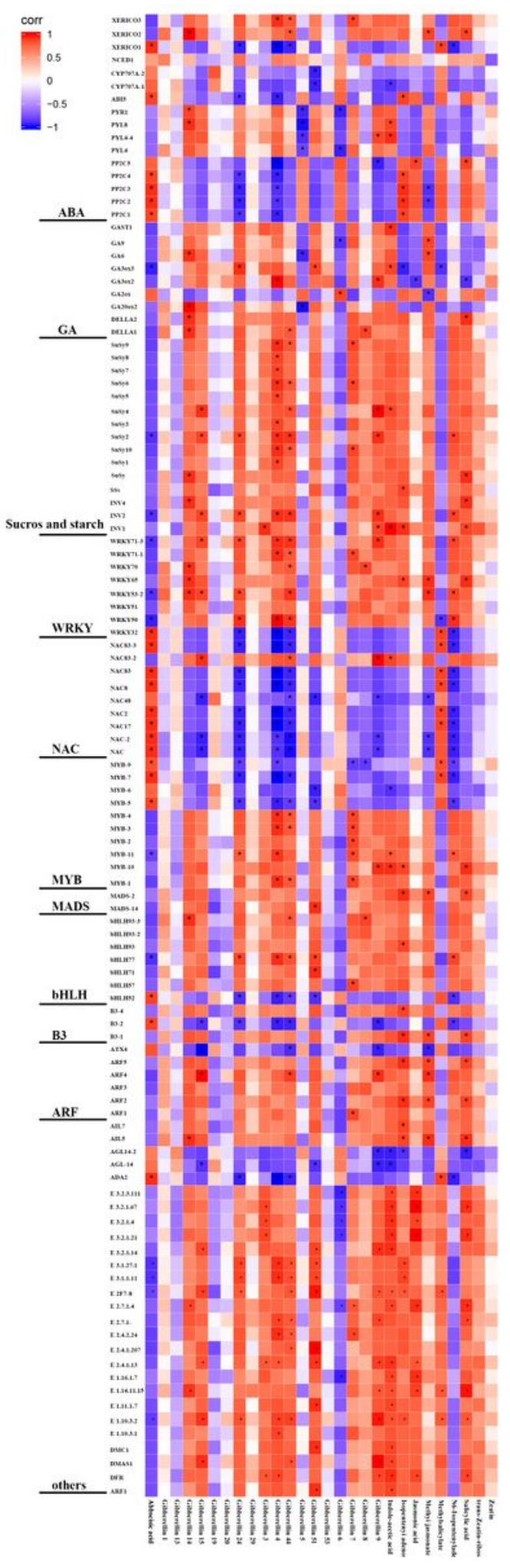

\section{Figure 6}

Heat map of the correlations between transcriptome and the targeted metabolome. The ordinate is the DEGs in the transcriptome, and the abscissa is the type of targeted metabolites. The red and blue represented the positive or negative correlation respectively. The * indicated that there is a significant correlation between genes and targeted metabolites ( $\mathrm{P} \mathrm{b} 0.05)$. 


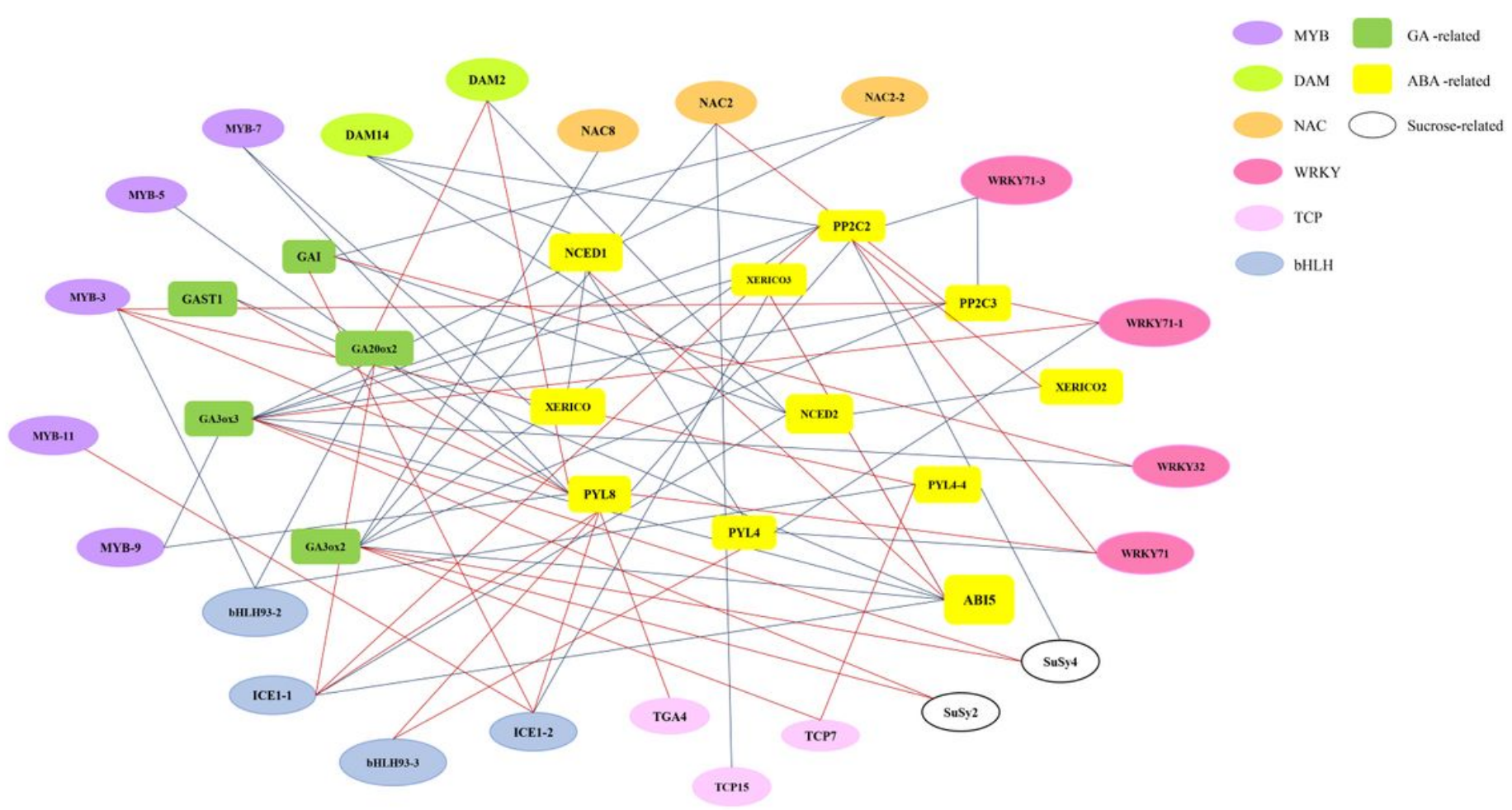

Figure 7

TFs and key DEGs correlation network. The red line indicates a positive correlation between the genes, and the blue line indicates the opposite. 


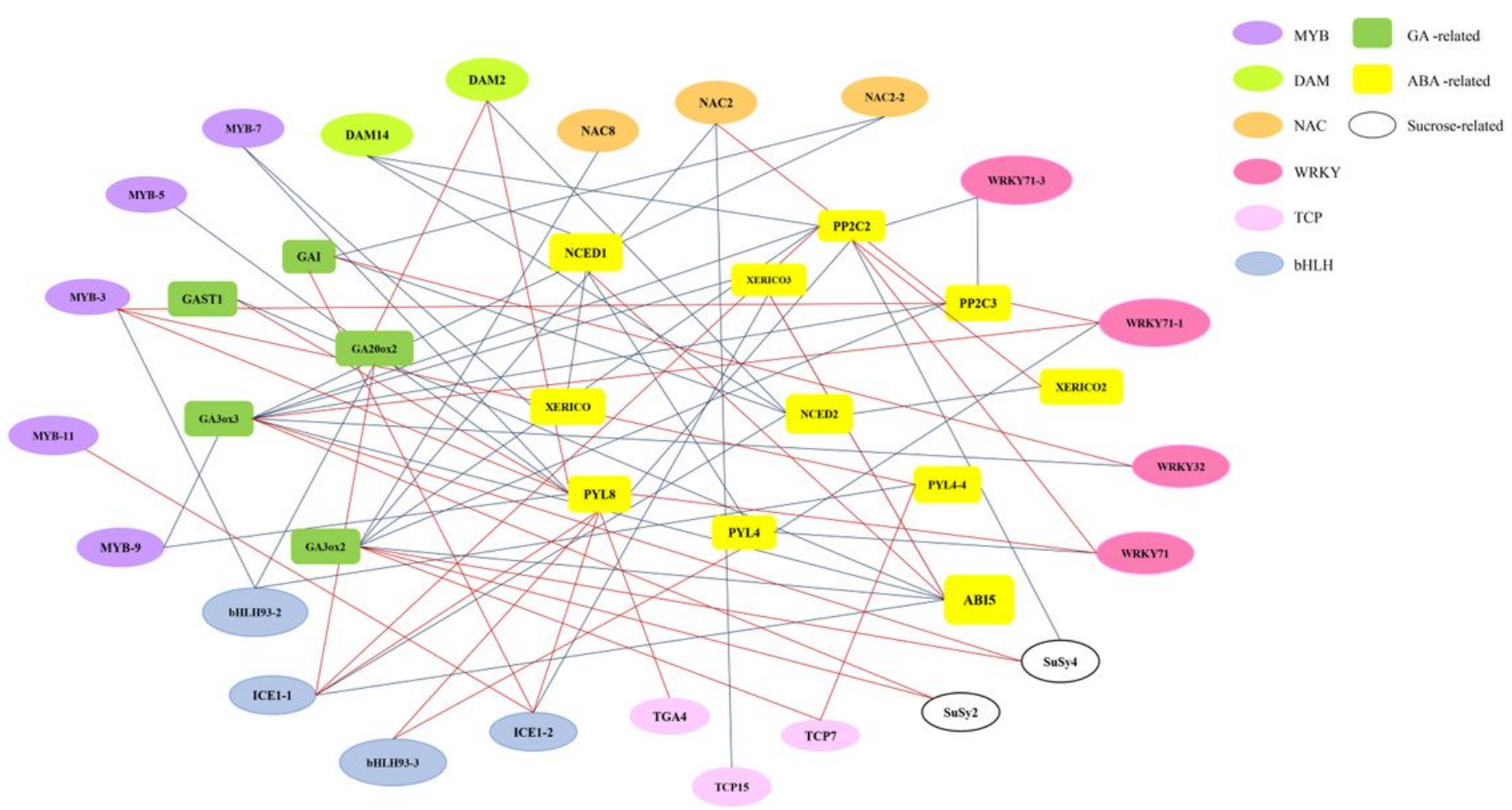

Figure 7

TFs and key DEGs correlation network. The red line indicates a positive correlation between the genes, and the blue line indicates the opposite. 
XERICO cluster-47386.19994

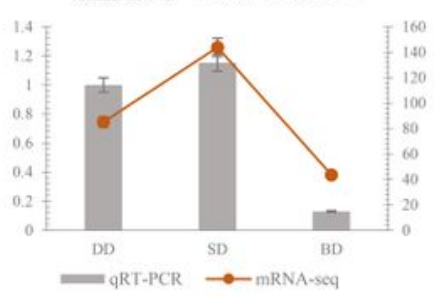

PYL4 cluster-47386.18322

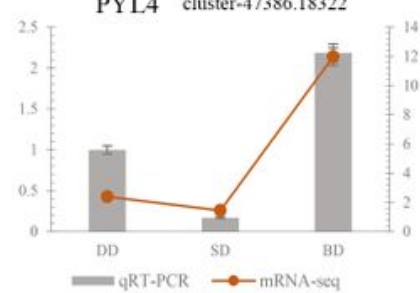

GA200X cluster-47386.44330

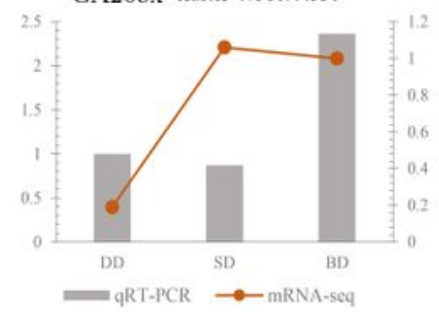

SuSy1 cluster-47386.21457

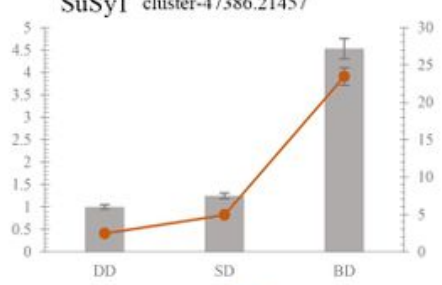

$\stackrel{\mathrm{DD}}{\longrightarrow} \mathrm{QRT} \cdot \mathrm{PCR} \longrightarrow \mathrm{mRNA}-\mathrm{sec}$

DAM14 cluster-47386.16917

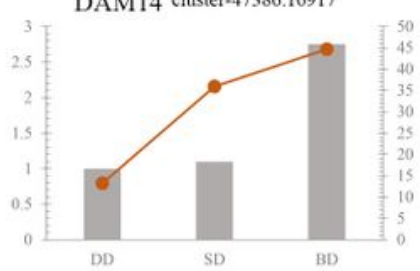

$\Longrightarrow$ qRT-PCR $\rightarrow$ mRNA-seq
NCED2 cluster-47386.6656

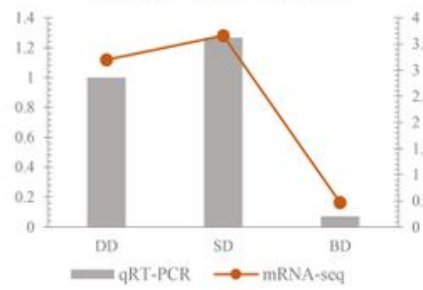

PP2C cluster-47386,47373

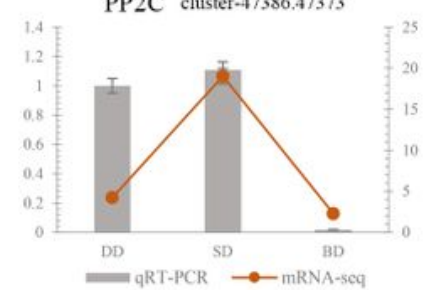

GA3ox cluster-47386.12758

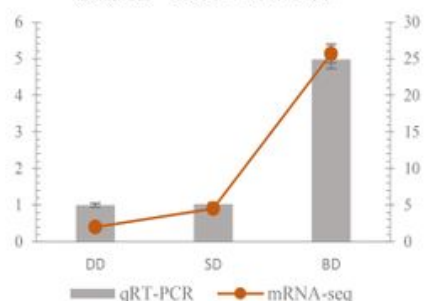

SuSy2 cluster-47386.29385

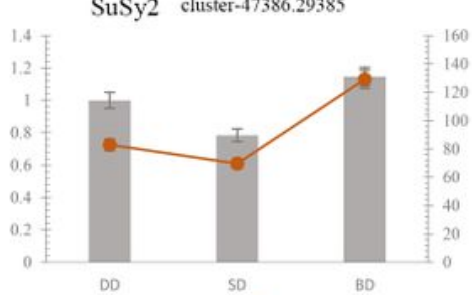

$\stackrel{\text { DD }}{=\text { QRT-PCR } \stackrel{\text { SD }}{\longrightarrow} \longrightarrow \text { mRNA-seq }}$

DAM2 cluster-47386.15589

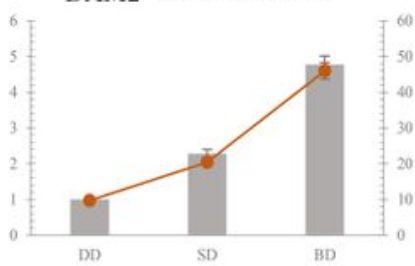

$\longrightarrow$ qRT-PCR $\rightarrow$ mRNA-seq
NCEDl cluster-47386.24866

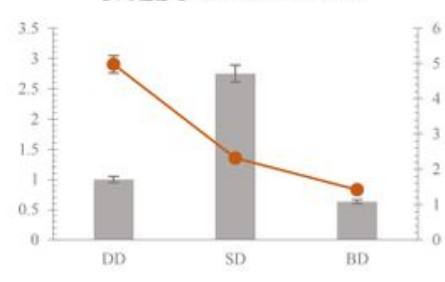

CYP707A cluster-47386.25413

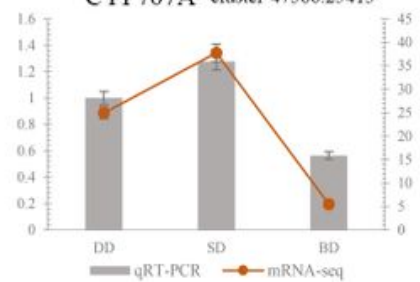

GAST1 cluster-47386.14948

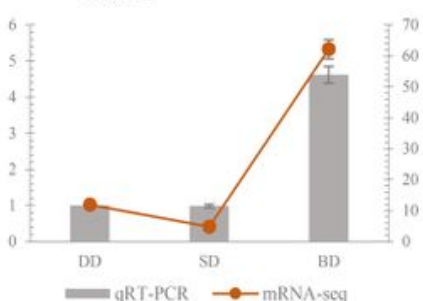

SuSy3 cluster-47386.29385

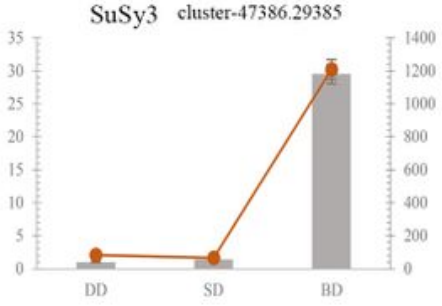

$\longrightarrow$ qRT.PCR $\rightarrow$ mRNA-seq

ICE1-2 cluster-47386.11386

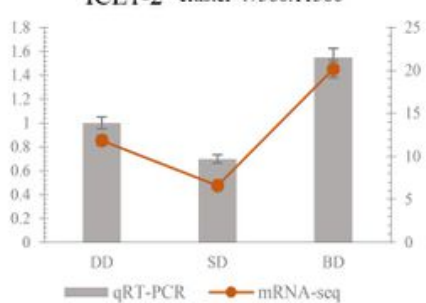

ABI5 cluster-47386.24673

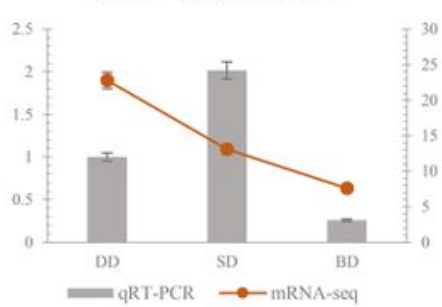

ABF2 cluster-47386.25413

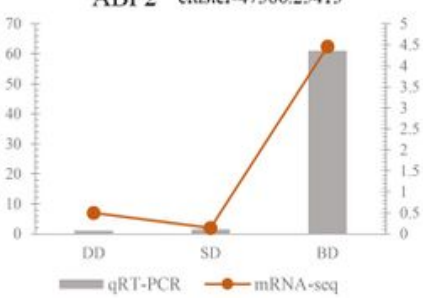

INV cluster-47386.41633

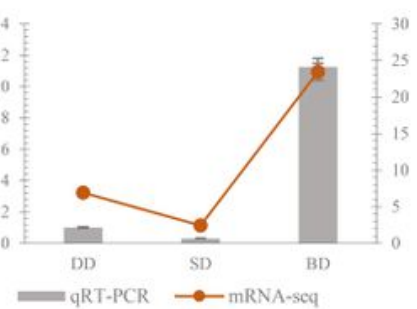

WRKY71 cluster-47386.23821

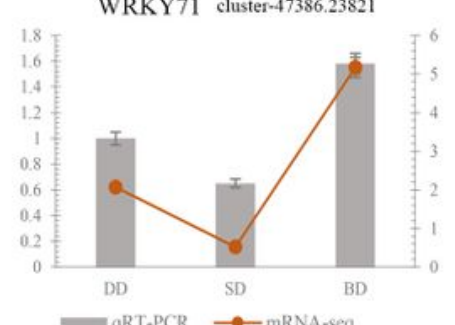

ICE1-1 cluster-47386.40624

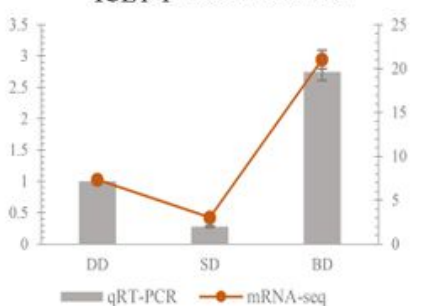

\section{Figure 8}

mRNA - seq and qRT-PCR values of 20 differentially expressed genes (DEGs). FPKM values were used for transcriptome data. Each set of experiments consisted of three biological and three technical replicates. 
XERICO cluster-47386.19994

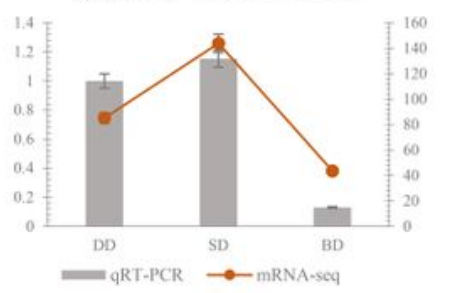

PYL4 cluster-47386.18322

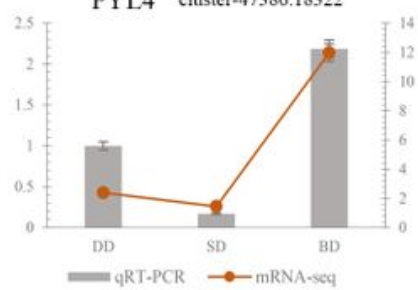

GA200X cluster-47386.44330

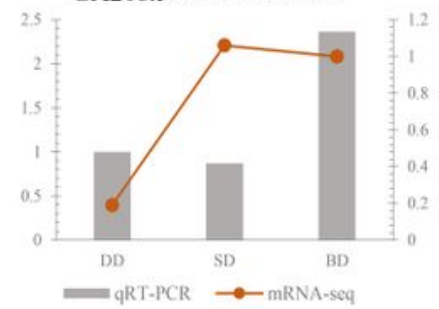

SuSy1 cluster-47386.21457

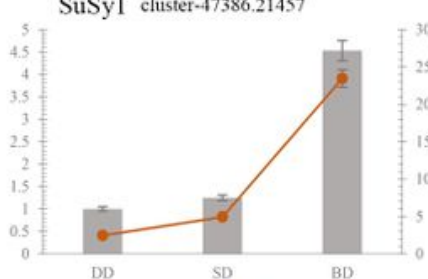

$\stackrel{\mathrm{DRT}}{\longrightarrow} \mathrm{PCR} \stackrel{\mathrm{mRNA}-\mathrm{seq}}{\longrightarrow}$

DAM14 cluster-47386.16917

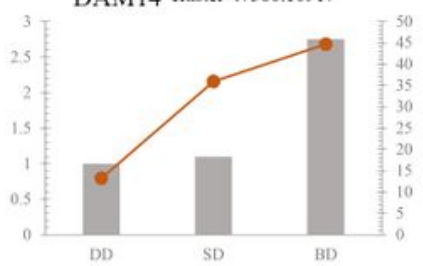

$\Longrightarrow$ QRT-PCR $\rightarrow$ mRNA-seq
NCED2 cluster-47386.6656

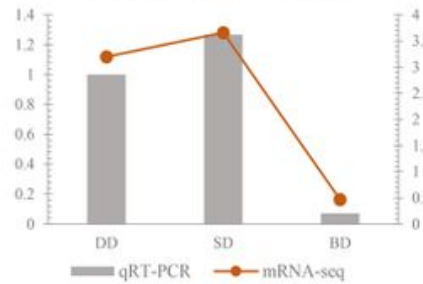

PP2C cluster-47386,47373

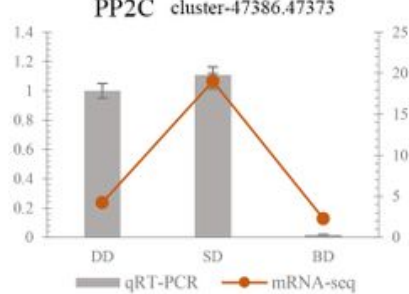

GA30X cluster-47386.12758

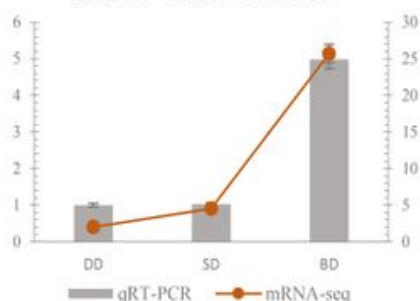

SuSy2 cluster-47386.29385

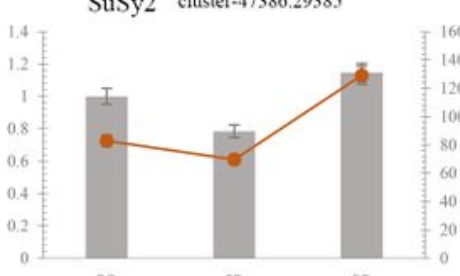

$\stackrel{\text { DD }}{=}$ QRT-PCR $\stackrel{\text { SD }}{\longrightarrow} \longrightarrow$ mRNA-seq

DAM2 cluster-47386.15589

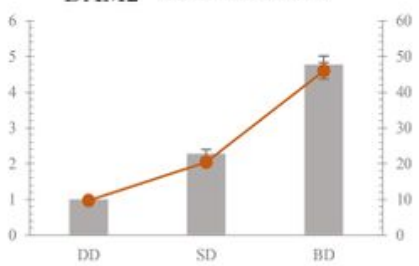

$\longrightarrow$ qRT-PCR $\rightarrow$ mRNA-seq
NCEDl cluster-47386.24866

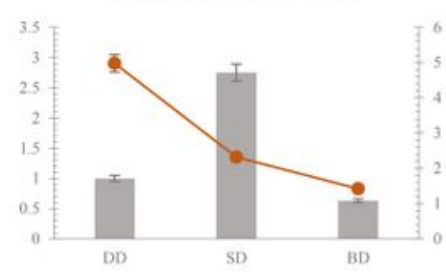

CYP707A cluster-47386.25413

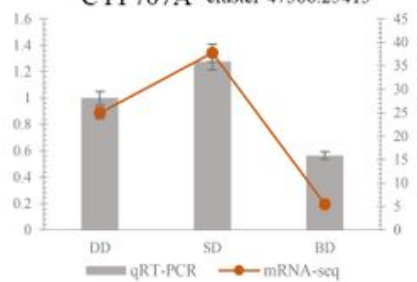

GAST1 cluster-47386.14948

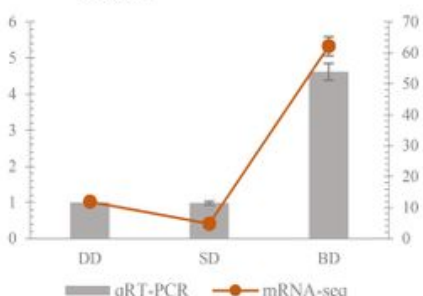

$\longrightarrow$ qRT.PCR $\rightarrow$ mRNA-seq

SuSy3 cluster-47386.29385

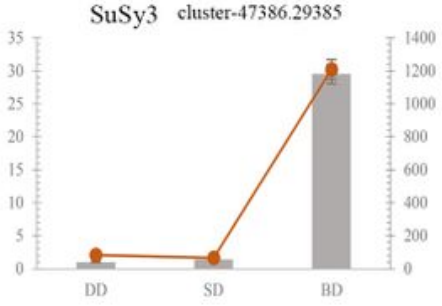

$\longrightarrow$ qRT.PCR $\rightarrow$ mRNA-seq

ICE1-2 cluster-47386.11386

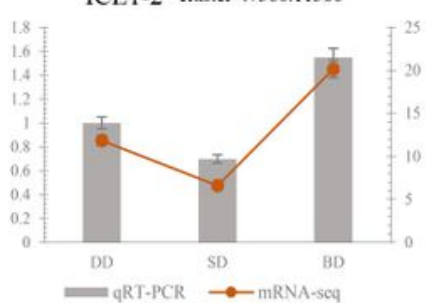

ABI5 cluster-47386.24673

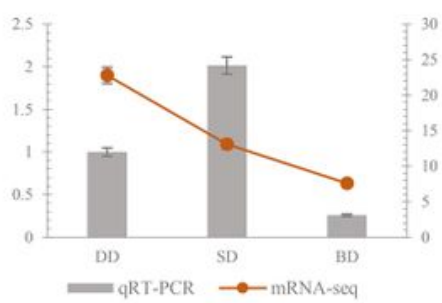

ABF2 cluster-47386.25413

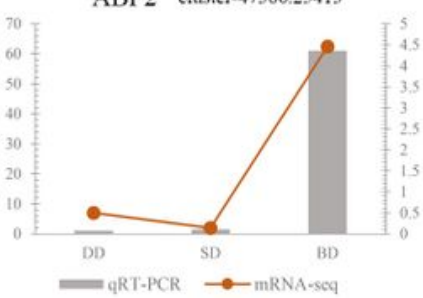

INV cluster-47386.41633

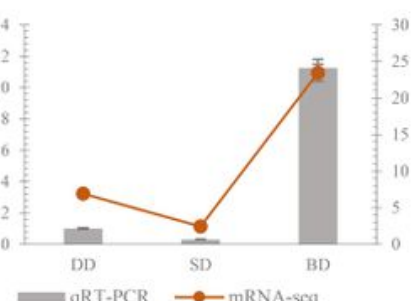

WRKY71 cluster-47386.23821

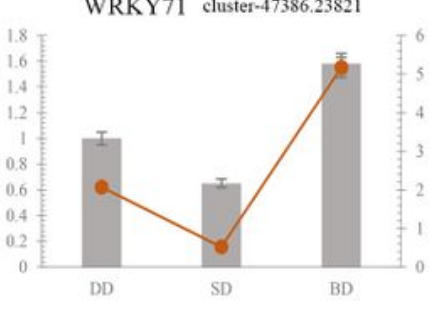

qRT-PCR $\rightarrow$ mRNA-seq

ICE1-1 cluster-47386.40624

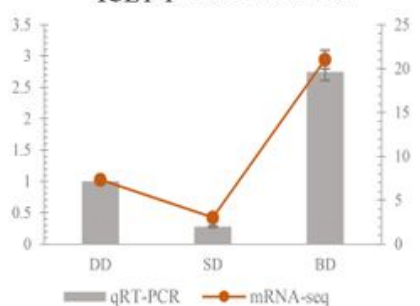

\section{Figure 8}

mRNA - seq and qRT-PCR values of 20 differentially expressed genes (DEGs). FPKM values were used for transcriptome data. Each set of experiments consisted of three biological and three technical replicates.

\section{Supplementary Files}


This is a list of supplementary files associated with this preprint. Click to download.

- Additionalfile1TableS1.docx

- Additionalfile1Tables1.docx

- Additionalfile2Fig.S1.jpg

- Additionalfile2Fig.S1.jpg

- Additionalfile3Fig.S2.jpg

- Additionalfile3Fig.S2.jpg 